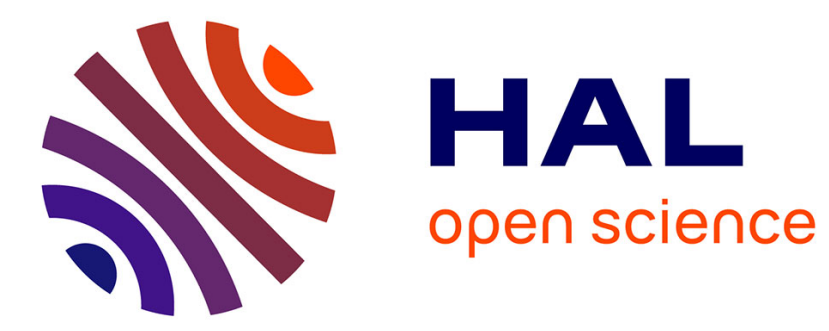

\title{
Boundary effects in large deviation problems
}

\author{
I.A. Ignatyuk, Vadim A. A. Malyshev, V.V. Scherbakov
}

\section{To cite this version:}

I.A. Ignatyuk, Vadim A. A. Malyshev, V.V. Scherbakov. Boundary effects in large deviation problems. [Research Report] RR-1999, INRIA. 1993. inria-00074673

\section{HAL Id: inria-00074673 https://hal.inria.fr/inria-00074673}

Submitted on 24 May 2006

HAL is a multi-disciplinary open access archive for the deposit and dissemination of scientific research documents, whether they are published or not. The documents may come from teaching and research institutions in France or abroad, or from public or private research centers.
L'archive ouverte pluridisciplinaire HAL, est destinée au dépôt et à la diffusion de documents scientifiques de niveau recherche, publiés ou non, émanant des établissements d'enseignement et de recherche français ou étrangers, des laboratoires publics ou privés. 


\title{
Boundary Effects in Large Deviation Problems
}

\author{
I.A. IGNATYUK \\ V. MALYSHEV \\ V.V. SCHERBAKOV
}

No 1999

Juillet 1993

PROGRAMME 1

Architectures parallèles,

bases de donnces,

réseaux et systèmes distribués

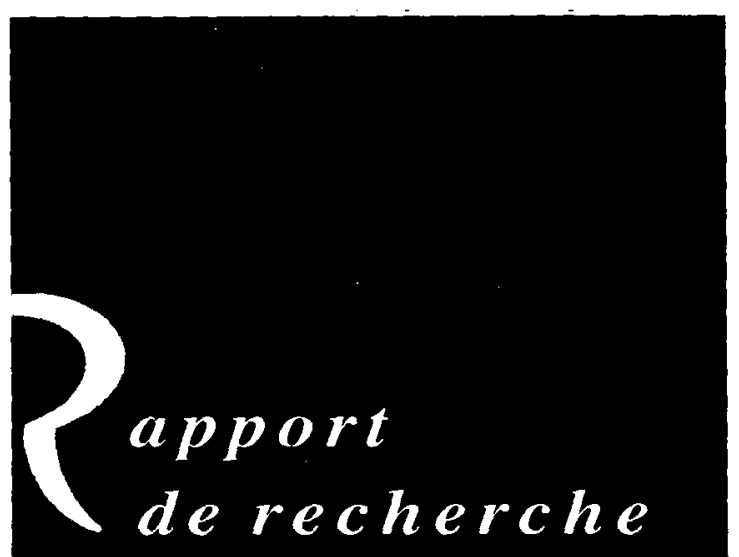




\title{
Effets de bord dans les problèmes de grandes déviations
}

\author{
I.A.Ignatyuk * V.Malyshev ${ }^{\dagger} \quad$ V.V.Scherbakov* \\ July 9, 1993
}

\begin{abstract}
A bstract
Nous obtenons des formules explicites pour les problèmes de grandes déviations sur $Z_{+}^{1} \cdot Z_{+}^{1} \times Z^{\mu}, Z^{\mu}$ muni d'une discontinuité planaire. et sur $Z_{+}^{2}$. C'es résultats inattendus sont obtenus à l'aide de nouvelles méthodes probabilistes assez simples.
\end{abstract}

- Postal address: Laboratory of Large Random Systems. Moscow State Iiniversity. Moscow. 119899). Russia

'Postal address: I.N.R.I.A.- Domaine de Voluceau- Rocquencourt-B.P.105-88153, Le Chesnay Colex.lature 


\title{
Boundary Effects in Large Deviation Problems
}

\author{
I.A.Ignatyul * V.Malyshev ${ }^{\dagger} \quad$ V.V.Scherbakov* \\ July 12,1993
}

\begin{abstract}
We get explicit formulas for large deviation problems in $Z_{+}^{1}, Z_{+}^{1} \times Z^{\mu}$. $Z^{\prime \prime}$ with a discontinuity along an hyperplane and on $Z_{+}^{2}$. These quite unexpected results are obtained by using new simple probabilistic technigues.
\end{abstract}

* Postal addrem: Laboratory of Large Random Systems, Moscow State University. Moscow, $119 x ! 99$. Russia

tPostal adrlress: I.N.R.I.A.- Domaine de Voluceau- Rocquencourt-B.P.105-7815;). Le Chesnay Ciedex. France 


\section{General introduction.}

Large deviation theory for Markor processes is now a well developed field with exccllent expositions (sce [ij. [\$]. et.c.) Nevertheless. this mainly concerns the cases where transition rates (or coefficients, for diffusion processes) vary continuously in space under appropriate scaling. We consider here some typical examples when there is a discontinuity with (generalised) reflection. Ir give the definition of (defined in [1]) deflected random walks $S(t) . t=0.1 \ldots$ in $Z_{+}^{\prime \prime}$. or more generally in $Z_{+}^{\nu} \times Z^{\mu}$. In the next sections we give separate definitions for all the examples we consider,so the rader can slip thic imroduction. Denote the points of $Z_{+}^{\prime \prime}$ by $x=\left(n_{1}, \ldots . n_{1}\right)$. Let.$\perp \subset\left\{1 \ldots \ldots\right.$, . Ne denote by .1 also the face $\left\{\left(n_{1} \ldots \ldots n_{\nu}\right): n_{i}>0 . i \in\right.$ $\left..1: n_{i}=0 . i \notin .1\right\}$. Define discrete time homogeneous Markov chain with state space $Z_{+}^{\prime \prime}$ and transition probabilitics $p_{x \cdot y}$, satisfying the following maximal homogeneit! condition :

$$
p_{x, y}=p(\Lambda ; y-x), x \in \Lambda
$$

That is. He transition probabilities depend only on the face to which $x$ belongs and also on the difference between the points.

We assmuc also boundedness of jumps : $p_{x . y} \neq 0$ can occur only if for all i we hav $-1 \leq y_{i}-r_{i} \leq d$ for some $d \geq 1$. Note that it is an exercise to generalise on results to the case when $d=\infty$ but all exponential moments exist.

The parameter space for these random walks is the direct product

$$
\mathcal{P}=\times \mathcal{F}_{\Lambda}
$$

(over all faces .1) of $P_{\Lambda}$. where $\mathcal{P}_{\Lambda}=\{p(\Lambda, \cdot)\}$ is the parameter space for the face 1 .

We comsiden large deviation problems for such random walks. Examples of such problems are the following:

(i) as!mptotics of $\log P(S(N)=[x N]), N \rightarrow \infty$ for some $x \in R_{+}^{\prime \prime}$;

(ii) logarithmic assmptotics of the stationary probabilities $\pi([x N])$ for ergodic deflected random walks: 
(iii) asymptotics of $\log P\left(\sup _{i \leq N \tau}\left|S(i)-N \varphi\left(\frac{i}{N}\right)\right|<\delta N\right)$ for some function $\varphi(t):[0, \tau] \rightarrow R_{+}^{u}$.

We shall see that example (iii) is the most general and (i) and (ii) appear as corollaries from (iii). Moreover, we shall see that it is sufficient to consider pjecewise linear functions $\varphi(t):[0, \tau] \rightarrow R_{+}^{\nu}$.

There are important results concerning these problems ( see for example [2] and [3]). However, we provide here a new insight to them. First of all we try (via a simple probabilistic approach and new tricks) to get the results as explicitely as possible using our experience (see $[1],[-1],[5])$ in studying deflected random walks in $Z_{+}^{\nu}$.

Secondly, we study an influence of the boundary parameters on the asymptotics. For example,we consider the following problems.

A. For which $x$ does the limit

$$
\lim _{N \rightarrow \infty} \frac{1}{N} \log \pi([N x])=l=l(x)
$$

not depend on the boundary jumps? More exactly. when does $l(x)$ not depend on parameters $\mathcal{F}_{\Lambda}, \Lambda \neq\{1, \ldots, \nu\}$ ?

B. We study "critical surfaces" in $\mathcal{P} \times\{x\}$, where $l$ is no more differentiable with respect to parameters, $l$ is smooth outside these critical surfaces.

We compare earlier analytic results for a quarter plane (see [6]) with our results. But we have not revealed deep connections between the analytic approach (through complex spaces) and probabilistic approach (through real space).

Similar to stability problems (see [1]) the complexity of large deviation problem strongly depends on the codimension of boundaries, i.e. on $\nu$. Here we consider codimension 1 and 2. For larger codimensions our methods also work in many particular cases but we have no a complete picture at the present moment.

To make the paper readable for beginners in large deviations we included an introductory section 2.1.

\section{Analytic methods.}




\subsection{Steepest descent and Legendre transform for sums of independent random variables.}

In this sulssection we give a brief introduction to the analytic methods for large deviation problems.

Let $S(t) . t=0,1, \ldots$, be a homogeneous random walk in $Z^{\prime \prime}$ with bounderl jumps, starting from 0 .

Consider $S(N)$ as the sum of i.i.d. random variables : $S(N)=\xi_{1}+\cdots+$ $\xi_{N}$.

Then we have

$$
P(S(N)=[x N])=\frac{1}{2 \pi i} \int_{|z|=1} \frac{1}{z^{[x N]+1}} E z^{S(N)} d z
$$

where

Put $z=\exp \lambda$ and note that $E^{S(N)}=(E \exp (\lambda \xi))^{N}=\exp (. H(\lambda))$.

$$
H(\lambda)=\log E \exp (\lambda \xi)
$$

is the semiinvariant generating function. Rewriting the integral (3) as

$$
\frac{1}{2 \pi i} \int_{|=|=1} \exp (N(H(\lambda)-x \lambda)) \exp \left(-\lambda+\epsilon_{N}\right) d z
$$

where $c_{N}=-\left[x N^{N}\right]+x N$, we want to use the method of steepest descent for this integral (5) and so we have to look for the critical points of the function $H(\lambda)-x \lambda$. The function $H(\lambda)$ is convex and analytic in the complex plane. so for $z>0$ the critical point $\lambda_{c r}=\lambda(x)$ is unique or none and we can use: the contour (when $\lambda_{i}$ exists)

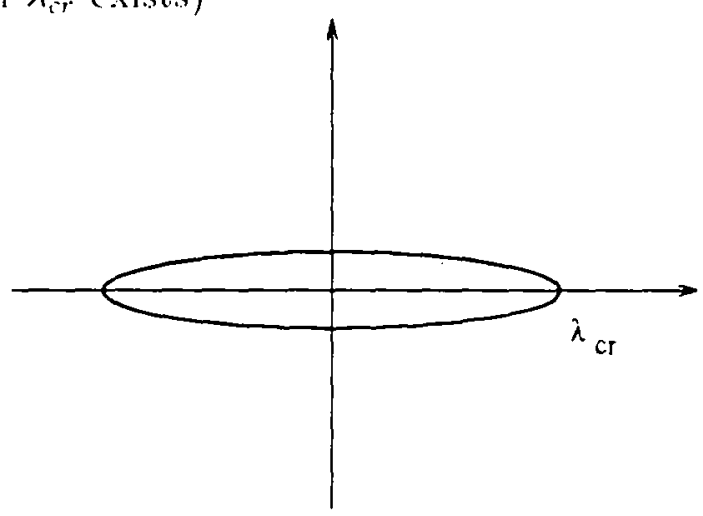


We are interested here only in the exponent in the integrand of (5):

$$
L(x)=-H(\lambda(x))+x \lambda(x)=\sup (-H(\lambda)+x \lambda)
$$

which is called the Legendre transform of $H(x)$. So the method of steepest clescent gives us the following result.

\section{Theorem 2.1.1}

$$
\log P(S(N)=[x N]) \sim-L(x) N
$$

$a s N \rightarrow \infty$

Let uniformly bounded $\xi_{t}$ be dependent but strongly mixing. for examplo assume them to be Gibbs with translation invariant two-point interaction

$$
l_{i}=\sum_{t, s} V(t-s) \xi_{t} \xi_{s}
$$

with exponential decrease. Then as it is well known the "partition function" $E \exp \left(\lambda S\left(\mathrm{~N}^{\prime}\right)\right)$. which is the ratio of two partition functions

$$
E \exp (\lambda S(N))=\frac{\sum_{\{\xi\}} \exp \left(-U+\lambda \sum_{t=1}^{N} \xi_{t}\right)}{\sum_{\{\xi\}} \exp (-U)}
$$

has the asymptotics

$$
\log E \exp (\lambda . S(N)) \sim N(H(U, \lambda)-H(U, 0))+\text { const }+o(1)
$$

where $H(l ; \lambda)$ is called the free energy of the "partition function" of the numerator. Moreover it is known to be analytic and convex for real $\lambda$. There are several methods (see [10], [11]) to prove all these assertions and calculate the frec energy in one dimension: renormalisation group with cluster expansions. transfer matrix method etc. They are applicable for all $\lambda$. So the theorem 2.1.1 holds for this case as well.For Markov chains (or when the interaction has a finite radius, to get a Markov chain we can assume the radius equal 1 )

$$
H(l ; \lambda)=\rho_{1}(l, \lambda)
$$

where $\rho_{1}(l, \lambda)$ is the maximal eigenvalue of the (positive) transfer-matrix $\left(p_{i, j} \exp (\lambda(j-i))\right)$ where $p_{i,}$ are the transition probabilities for the Markor chain $\xi_{t}$. Vote that $\rho_{1}(l .0)=1$. 


\subsection{Analytic methods in a quarter plane.}

In this subsection we formulate the main results of $[6]$ on the asymptotic behaviour of stationary probabilities for random walks in the quarter plane in terms of zeros of some simple polynomial equations in the complex plance.

Denote points of $Z_{+}^{2}$ by $(k, l)$ and consider a discrete time homogeneous Markov chain with this state space. Let $P\left((k, l) \rightarrow\left(k^{\prime}, l^{\prime}\right)\right)$ be its one step transition probabilities. Assume that they can be different from zero only if $-1 \leq k^{\prime}-k \leq d$ and $-1 \leq l^{\prime}-l \leq d$ for some $d>0$. Also assume homogeneity conditions (putting $i=k^{\prime}-k \cdot j=l^{\prime}-l$ )

$$
P\left((k . l) \rightarrow\left(k^{\prime} . l^{\prime}\right)\right)= \begin{cases}p_{i, j} & \text { if } k, l>0 \\ p_{i, j}^{\prime} & \text { if } l=0 . k>0 \\ p_{i, j}^{\prime \prime} & \text { if } l>0 . k=0 \\ p_{i, j}^{0} & \text { if } l=0, k=0\end{cases}
$$

We recall here the results of $[6]$ where it was assumed also that (i) $d=1$.

(ii) inside the quarter plane only the transition probabilities $p_{0.1}, p_{1,0}, p_{-1.0}, p_{0 .-1}$ are different from zero.

(iii) components of the inside mean jump vector

$$
M=\left(. M_{1} . . M_{2}\right)=\left(\sum_{i, j} i p_{i j}, \sum_{i, j} j p_{i j}\right)
$$

are negative. and

$$
\sum_{i} p_{i 1}^{\prime} \neq 0 . \sum_{j} p_{1 j}^{\prime \prime} \neq 0
$$

Assumption (ii) is not crucial for the applicability of the analytical methods but simplifies computations considerably. Besides the case (iii) there is also the case when only one of $M_{1} . M_{2}$ is negative. This case can be considered similarly:

Introduce the polynomial generating functions

$$
\begin{aligned}
& Q(x, y)=x y\left(1-\sum_{i, j} p_{i, j} x^{i} y^{j}\right) . \\
& q(x, y)=x\left(\sum_{i, j} p_{i, j}^{\prime} x^{i} y^{j}-1\right) .
\end{aligned}
$$




$$
q^{\prime}(x, y)=y\left(\sum_{i, j} p_{i, j}^{\prime \prime} x^{i} y^{j}-1\right)
$$

Consider the Riemann surface $\mathcal{S}$ of the algebraic functions $y(x)$ (or $x(y)$ ) defined by the equation

$$
Q(x, y)=0
$$

Let $x(s), y(s)$ be meromorphic functions on $S$ defining the coverings of the $x$-planc and $y$-plane respectively of 5 . We formulate here some results from $[6]$

1. There are exactly four branching points $x_{i}$ of the algebraic function $y(x)$ (or of $x^{-1}: 0<x_{1}<x_{2}<1<x_{3}<x_{4}$. Similarly for $x(y)$.

2. We call,$\%=\{s: r(s)$ and $y(s)$ are real $\}$ the set of real points of 5. S. consists of two disjoint analytic closed curves homologous to one of the elements of the nomal homology basis on $S$ (more exactly to the one different from $x^{-1}(\{x:|x|=1\})$. One of them, call it $F_{0}$. has the property that on it: $x_{2} \leq x(s) \leq x_{3} \cdot y_{2} \leq y(s) \leq y_{3}$. Denote the other one by $F_{1}$. $F_{u}$ has an ordered set of eight characteristic points $s_{0}, \ldots s_{7}$.

We show circles on the $x$-plane and on the $y$-plane : point $s_{0}=(1,1)$ is indicated and the arrows show the further correspondence between the values of $x\left(s_{i}\right)$ and $y\left(s_{i}\right)$.

$$
\begin{gathered}
x\left(s_{i}\right)=x_{2} \cdot x\left(s_{i}\right)=x\left(s_{0}\right)=1 . x\left(s_{5}\right)=x\left(s_{1}\right)=\sqrt{\frac{p_{0,-1}}{p_{0.1}}} \cdot x\left(s_{4}\right)=x\left(s_{2}\right)=\frac{p_{0,-1}}{p_{0.1}} \cdot x\left(s_{3}\right)=x_{3} \\
y\left(s_{i}\right)=y_{2} \cdot y\left(s_{i j}\right)=y\left(s_{0}\right)=1 . y\left(s_{5}\right)=y\left(s_{1}\right)=\sqrt{\frac{p_{-1.0}}{p_{1.0}}} \cdot y\left(s_{4}\right)=y\left(s_{2}\right)=\frac{p_{-1.0}}{p_{1.0}} \cdot y\left(s_{3}\right)=y_{3} \\
\hline
\end{gathered}
$$

3. The finction $0_{-}(s)=|x y| .0 \leq \gamma<1$. has (in the domain $0_{\gamma}^{-1}((0, x))$ ) four nondegencrate critical points $s_{i}(\gamma), i=1 \ldots . .4$. Any $s_{i}(\gamma)$ continuously (copends onl ; that muicquely defines these points if we agree that $x\left(s_{i}(0)\right)=x_{1}$. 
Moreover, $s_{2}(\gamma), s_{3}(\gamma) \in F_{0}$ and $x_{i}(\gamma)=x\left(s_{i}(\gamma)\right), y_{i}(\gamma)=y\left(s_{i}(\gamma)\right)$ are ral. For $\gamma=1$. one can put $s_{1}(1)=0, s_{4}(1)=\infty$, and for the critical points $s_{2}(1) . s_{3}(1)$ the above assertions hold. Equations defining these critical points are

$$
Q(x, y)=0
$$

and

$$
\frac{y}{\gamma x}=\frac{\frac{d}{d x} Q(x, y)}{\frac{d}{d y} Q(x, y)}
$$

We have also

$$
\begin{aligned}
& 1<x_{3}(1)<x_{3}(\gamma)<x_{3}(0)=x_{3} \\
& y_{3}(0)=\sqrt{\frac{p_{0,-1}}{p_{0,1}}}<y_{3}(\gamma)<y_{3}(\gamma) .
\end{aligned}
$$

It appears that the asymptotics of the stationary probabilities is clefined either by the critical points $s_{3}(\gamma)$ or by the poles in the points $s_{0}(\gamma)$ and $s_{u}^{\prime}(\gamma)$ where correspondingly $q\left(\zeta s_{0}\right)=0$ or $q^{\prime}\left(\eta s_{0}^{\prime}\right)=0$. The Cialois automorphisms $\zeta$ and $\eta$ on $s$ are defined by

$$
\zeta(x, y)=\left(x, \frac{p_{0 .-1}}{p_{0,1} y}\right), \eta(x, y)=\left(\frac{p_{-1,0}}{p_{1.0} x}, y\right)
$$

In the parameter space $\mathcal{P} \times\{\gamma: 0<\gamma \leq 1\}$ define the subsets

$$
\begin{aligned}
& \mathcal{F}_{--}=\left\{(p, \gamma): q\left(x_{3}(\gamma), \frac{p_{0,-1}}{p_{0,1} y_{3}(\gamma)}\right) \leq 0, q^{\prime}\left(\frac{p_{-1.0}}{p_{1.0} x_{3}(\gamma)} \cdot y_{3}(\gamma)\right) \leq 0\right\}, \\
& \mathcal{F}_{+-}=\left\{\left(p, \gamma_{i}\right): q\left(x_{3}(\gamma), \frac{p_{0,-1}}{p_{0.1} y_{3}(\gamma)}\right)>0, q^{\prime}\left(\frac{p_{-1.0}}{p_{1.0} \cdot r_{3}(\gamma)}, y_{3}(\gamma)\right) \leq 0\right\}
\end{aligned}
$$

and $\mathcal{P}_{-+} \cdot \mathcal{P}_{++}$correspondingly. 
Theorem 2.2.1 (Sec [6]). Let $m, n \rightarrow \infty$ so that $\frac{n}{m}=\gamma$. Then in $\mathcal{P}_{--}$u'e have

$$
\pi(m . n) \sim \frac{\text { const }}{\sqrt{m}}\left(r_{3}(\gamma) y_{3}(\gamma)\right)^{-m}
$$

Otherwise

$$
\pi(m . n) \sim \begin{cases}\operatorname{const}\left(x_{0}(\gamma) y_{0}^{\gamma}(\gamma)\right)^{-m} & \text { in } \mathcal{P}_{-+} \\ \operatorname{const}\left(x_{5}(\gamma) y_{5}^{\gamma}(\gamma)\right)^{-m} & \text { in } \mathcal{F}_{+-} \\ \operatorname{const}\left(x_{0}(\gamma) y_{0}^{\gamma}(\gamma)\right)^{-m}+\operatorname{const}\left(x_{5}(\gamma) y_{5}^{\gamma}(\gamma)\right)^{-m} & \text { in } \mathcal{F}_{++}\end{cases}
$$

where $1<x_{0}(\gamma)<x_{3}(\gamma) .1<y_{0}(\gamma)<\frac{p_{0,-1}}{p_{0,1} y_{3}(\gamma)}$ and $1<x_{5}(\gamma)<\frac{p-1.0}{p_{1.0} x_{2}(\gamma)}, 1<$ $y_{3}\left(\gamma_{i}\right)<y_{3}\left(\gamma_{i}\right)$ are defined from the systems

$$
\begin{aligned}
& Q(x, y)=0, q(x, \zeta y)=0 \\
& Q(x, y)=0, q^{\prime}(\eta x, y)=0
\end{aligned}
$$

correspondingly.

\section{Probabilistic method.}

\subsection{General definitions.}

The main goal of this paper is to prove the large deviation principle and to find explicitely the action functionals for the class of random walks defined below. This large deviation principle will be used to get asymptotics of the stationary probabilities in a quarter plane.

Random walks We consider the random walk $S_{t}(x)$ in $Z_{+}^{\nu} \times Z^{\mu}$ starting at the point $x$. Let $A \subset\{1, \ldots, \nu\}$. We denote by $\Lambda$ also the face $\left\{\left(x_{1} \ldots \ldots x_{1}\right): x_{i}>0 . i \in \Lambda ; x_{i}=0, i \notin \Lambda\right\} \times R^{\mu} \subset R_{+}^{\prime \prime} \times R^{\mu}$. Define discrete time homogeneous Narkov chain with the state space $Z_{+}^{\prime \prime} \times Z^{\mu}$ and transition probabilities $p_{r . y}$. satisfying the following maximal homogencity condition :

$$
p_{x, y}=p(\Lambda: y-x), x \in \Lambda
$$


That is the transition probabilities depend only on the face to which er belongs and on the difference between the points.

We assume also boundedness of jumps : $p_{x, y} \neq 0$ can occur only if for all $i=1, \ldots, v$ we have for the $R_{+}^{\prime \prime}$-components : $-1 \leq y_{i}-x_{i} \leq d$ and for $R^{\mu}$-components $-d \leq y_{i}-x_{i} \leq d$ for some $d \geq 1$.

Large deviation principle For any $\tau \in R_{+}$consider the set $C\left([0, \tau], R_{+}^{\prime \prime} \times\right.$ $R^{\mu}$ ) of all continuous functions $\varphi:[0 . \tau] \rightarrow R_{+}^{\nu} \times R^{\mu}$. Let be given functionals $\mathcal{L}_{\tau}$. mapping the space $C\left([0 . \tau], R_{+}^{\prime \prime} \times R^{\mu}\right)$ into $[0 .+\infty]$. Consider for any: $s \geq 0$ and for any $x \in R_{+}^{\prime \prime} \times R^{\mu}$ the set

$$
\Phi_{x . r}(s)=\left\{r \in C\left([0 . \tau] . R_{+}^{\prime \prime} \times R^{\mu}\right): \varphi(0)=x \text { and } \dot{\mathcal{L}}_{r}(\varphi) \leq s\right\}
$$

Definition 3.1.1 His say that the random walk $S_{t}$ satisfies the large deviation principle with action functionals $\mathcal{L}_{\tau}$ if for any $\tau \geq 0$ and for any $x \in R_{+}^{\prime} \times R^{\prime \prime}$ the follouing three conditions hold.

(i) (compucturs.s.) For any $s \geq 0$ the set $\Phi_{x, \tau}(s)$ is compact.

(ii) (large deriation louer bound.) For any $\delta>0 . s_{0}>0, \delta^{\prime}>0$ there exists $N_{0}$ surlh that for an!y $N>N_{0}, \hat{i} \in \Phi_{x . \tau}\left(s_{0}\right)$. the following estimate holds

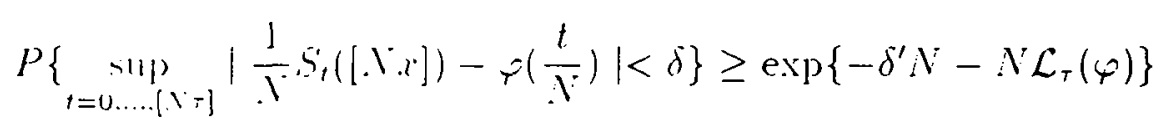

(iii) (large de ciation upper bound.)For any $\delta>0$. $\delta^{\prime}>0$, so $>0$, there exists $N_{0}$ such that for an!y $N>N_{0} .0<s<s_{0}$ the following estimate holds

$$
\begin{aligned}
& P\left\{\underset{t=0 \ldots \ldots\left[N_{T}\right]}{\operatorname{su}],}\left|\frac{1}{N} S_{t}([N x])-\varphi\left(\frac{t}{N}\right)\right| \geq \delta \text { for any } \varphi \in \Phi_{x, T}(s)\right\} \leq \\
& \leq \exp \left\{\delta^{\prime} N-N s\right\}
\end{aligned}
$$

Let us note that. due to the boundedness of the jumps of the random walk $S_{t}$. for any path $\varphi \in C^{\prime}\left([0 . \tau] . R_{+}^{\nu} \times R^{\mu}\right)$ (and even for discontinuous $\left.\varphi^{\prime}\right)$ for which hhere exist $0<t<t^{\prime}<\tau$ such that

$$
\left|\gamma(t)-y\left(t^{\prime}\right)\right|>d\left(t^{\prime}-t\right)
$$

we have

$$
P\left\{\sup _{t=0 \ldots \ldots\left[N^{\prime} \tau\right]}\left|\frac{1}{N} S_{t}([N x])-\varphi\left(\frac{t}{N^{\prime}}\right)\right|<\delta\right\}=0
$$


for sufficicntly small $\delta>0$ and sufficiently large $N$, and consequently if the random walk satisfies the large deviation principle with the action functionals $\mathcal{L}_{\tau}$ then for such $\tau$

$$
\mathcal{L}_{T}(\varphi)=+\infty
$$

\section{Lagrangians}

In the next sections we shall prove the large deviation principle for linear paths (in a stronger version, see conditions (ii) and (iii) of Theorem 3.1.1). We shall find also explicit expressions of the action functional for linear paths. We shall sec that it will have the following form.

Let for any face $A$ of $R^{\nu}$ a function

$$
L(\cdot A): R^{k} \times R^{\mu} \rightarrow R_{+} \bigcup\{+\infty\}
$$

be defined. Where $k$ is the dimension of $\Lambda$.Define the lagrangian. i.e. the function $L:\left(R_{+}^{\prime \prime} \times R^{\mu}\right) \times R^{\nu+\mu} \rightarrow R_{+} \bigcup\{+\infty\}$ such that for all $\Lambda$ and for all $x \in \Lambda$

$$
L(x, v)=L\left(v_{1}: \Lambda\right)
$$

where $v_{\Lambda}$ is the projection of $v$ onto $\Lambda$.

For a linear path $\tau^{2}:[0, \tau] \rightarrow R_{+}^{\prime \prime} \times R^{\mu}$ define its speed vector

$$
\because(\varphi)=\frac{\varphi(\tau)-\varphi(0)}{\tau}
$$

and the face $\Lambda=\Lambda(\nu)$ to which this path belongs. Then we shall prove that $\mathcal{L}_{\tau}(\varphi)=\tau L_{\Lambda(*)}((\varphi))$. In other words to find the action functional we should find the constants $L(A, v)$.

To satisfy condition ( $\mathrm{jv}$ ) of theorem 3.1.1 we should. for any $\tau>0$. define the functional

$$
\dot{L}_{T}(\hat{r})=\int_{0}^{T} L(\varphi(t) \cdot \dot{\varphi}(t)) d t .
$$

if $\varphi$ is absolutely continuous. and

$$
\dot{\mathcal{L}}_{\tau}(\varphi)=+\infty
$$

otherwise. Then from nice properties of the functions $L\left(.1, v^{*}\right)$ the conditions (i) and (iv) of theorem 3.1.1 will follow.

From linear to arbitrary paths 
Theorem 3.1.1 Let for the random walk s, the functionals $\mathcal{L}_{\tau}$ be giren such that for an! $\tau>0$ the following conditions are satisfied:

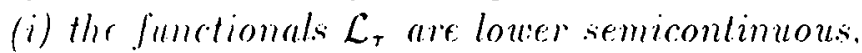

(ii) for any $\delta>0 . \delta^{\prime}>0$ one can find $\sigma>0$ such that for amy $x . y \in$ $R_{+}^{\prime} \times R^{\prime \prime}$ such that $|x-y|<\sigma$ and for any linear path $\varphi_{0} \in \Phi_{x . \tau}$ the following cstimate lolds:

$$
P\left\{\sup _{t=0 \ldots \ldots[N \tau]}\left|\frac{1}{N} S_{t}\left(\left[N^{r} y\right]\right)-\varphi\left(\frac{t}{N}\right)\right|<\delta\right\} \geq \exp \left\{-\delta^{\prime} N-N \mathcal{L}_{\tau}(\tau)\right\}
$$

for all sufficionlly large $N$.

(iii) for any $\delta^{\prime}>0$ one. can find $\delta>0, \sigma>0$ such that for any $x . y \in$ $R_{+}^{\prime \prime} \times R^{\prime \prime}$ surh that $|x-y|<\sigma$ and for any linear path $\varphi \in \Phi_{\text {r.t }}$ the following estimate holds.

$$
P\left\{\underset{t=0 \ldots \ldots \mid N \tau]}{\sup }\left|\frac{1}{N} S_{t}([N y])-\varphi\left(\frac{t}{N}\right)\right|<\delta\right\} \leq \exp \left\{+\delta^{\prime} N-N \mathcal{L}_{\tau}(\varphi)\right\}
$$

for all sulficiently large $N$.

(iv) for any $x \in R_{+}^{\prime} \times R^{\mu}, \varphi \in \Phi_{x, \tau}$ and for any $\epsilon>0$ there exists a pieceswise limfar path $\dot{\varphi} \in \Phi_{x, \tau}$ such that

$$
\sup _{0 \leq t \leq \tau}|\varphi(t)-\dot{\varphi}(t)|<\epsilon \text { and }\left|\mathcal{L}_{\tau}(\varphi)-\mathcal{L}_{\tau}(\dot{\varphi})\right|<\epsilon
$$

Then the rundom walk satisfies the large deviation principle with the action functionals $\dot{\mathcal{L}}_{\tau}$.

Proof. Denote by $\Phi_{r . \tau}$ the set of all continuous paths $\varphi:[0 . \tau] \rightarrow R_{+}^{\prime \prime} \times R^{\prime \prime}$ for which $\mathcal{L}_{T}(\varphi)<\infty$. Since for any $\varphi \in \Phi_{2 . \tau}$

$$
\sup _{0 \leq t<t^{\prime} \leq t} \frac{\left|\varphi(t)-\varphi\left(t^{\prime}\right)\right|}{t^{\prime}-t} \leq d
$$

then the scr. $\Phi_{x, \tau}$ is relatively compact. So if the for any $\tau>0$ the functional $\dot{L}_{\tau}$ is lower semicontimuous then the sets $\Phi_{x . \tau}(s)$ are compact.

To get large deviations bounds we go first from linear paths to piecewise linear and then to arbitrary paths.

For any piecewise linear path $y:[0, \tau] \rightarrow R_{+}^{\prime \prime} \times R^{\prime \prime}$ consider its linurar pieces pl $_{1}$.... it: 
let $0=\tau_{0}<\tau_{1}<\ldots<\tau_{k-1}<\tau_{k}=\tau$ and let $\varphi$ be linear on each interval $\left(\tau_{j}, \tau_{j+1}\right), j=0 \ldots, k-1$ then

$$
\hat{\tau, j}(t)=\varphi\left(t+\tau_{j}\right) .0 \leq t \leq \tau_{j+1}-\tau_{j}
$$

Lemma 3.1.1 let for any $\tau \geq 0, x \in R_{+}^{\nu} \times R^{\mu}$ and for any linear path $p \in \Phi_{x . r}$ the following two conditions hold:

(i) for any $\delta>0 . \delta^{\prime}>0$ one can find $\sigma>0$ such that for any $y \in R_{+}^{\prime \prime} \times R^{\prime \prime}$ such that $|x-y|<\sigma$ the following estimate holds

$$
P\left\{\underset{t=0 \ldots \ldots(N)]}{\sup }\left|\frac{1}{N} s_{t}([N y])-\varphi\left(\frac{t}{N}\right)\right|<\delta\right\} \geq \exp \left\{-\delta^{\prime} N-N \mathcal{L}_{\tau}(\varphi)\right\}
$$

for all sufficifntly lange $N$.

(ii) for any $\delta^{\prime}>0$ one can find $\delta>0, \sigma>0$ such that for any $y \in R_{+}^{\prime \prime} \times R^{\mu}$ such that $|x-y|<\sigma$ the follouing estimate holds

$$
P\left\{\sup _{t=0 \ldots \ldots[N]}\left|\frac{1}{N} S_{t}(\{N y])-\varphi\left(\frac{t}{N}\right)\right|<\delta\right\} \leq \exp \left\{+\delta^{\prime} N-N \mathcal{L}_{\tau}(\varphi)\right\}
$$

for all sufficiently large $N$.

Then for any $\tau>0$ the following conclusions hold

(ii) for any $\delta>0, \delta^{\prime}>0$ one can find $\sigma>0$ such that for any $x . y \in$ $R_{+}^{\nu} \times R^{\prime \prime}$ such that $|x-y|<\sigma$ and for any piecewise linear path $\varphi \in \Phi_{2, \tau}$ the following stimate holds

$$
P\left\{\operatorname{sil}_{t=0 \ldots \ldots\left[N^{\prime} \tau\right]}\left|\frac{1}{N} S_{t}([N y])-\varphi\left(\frac{t}{N}\right)\right|<\delta\right\} \geq \exp \left\{-\delta^{\prime} N-N \mathcal{L}_{\tau}(\varphi)\right\}
$$

for all sufficiently large $N$

(iii) for ally $\delta^{\prime}>0$ one can find $\delta>0, \sigma>0$ such that for any $x, y \in$ $R_{+}^{\prime \prime} \times R^{\prime \prime}$ such that $|x-y|<\sigma$ and for any piecewise linear path $\varphi \in \Phi_{x, \tau}$ the follouing fstimate holds

$$
P\left\{\sup _{i=0 \ldots[N \tau]}\left|\frac{1}{N} S_{t}([N y])-\varphi\left(\frac{t}{N}\right)\right|<\delta\right\} \leq \exp \left\{+\delta^{\prime} N-N \mathcal{L}_{\tau}(\varphi)\right\}
$$

for all surfficirmlly large $N$

with lle ardion functional

$$
\mathcal{L}_{\tau}(\varphi)=\sum_{j=1}^{k} \mathcal{L}_{\tau_{j+1}-\tau_{j}}\left(\varphi_{j}\right)
$$

when $+1 . . . .+\%$ are the linear pieces of $\varphi$. 
One can easily prove this lemma using the induction with respect to the number of linear pieces.

Lemma 3.1.2 Let for any $\tau \geq 0, x \in R_{+}^{\nu} \times R^{\mu}$ for any $\varphi \in \Phi_{x, \tau}$ and for any $\epsilon>0$ there exists a piecesuise linear path $\dot{p} \in \Phi_{x, \tau}$ such that

$$
\sup _{0 \leq 1 \leq \tau}|\hat{r}(t)-\dot{\varphi}(t)|<\epsilon \text { and }\left|\mathcal{L}_{\tau}(\varphi)-\mathcal{L}_{\tau}(\dot{\varphi})\right|<\epsilon
$$

and let for any $\tau>0$ and for any piccesuise linear path $\varphi \in \Phi_{x . \tau}$ lower laryf deviation bound $(18)$ and upper large deviation bound (19) hold.

Then lower lange deviation bound (14) and upper large deviation bound (15) hold.

Proof. Let $x \in R_{+}^{\prime \prime} \times R^{\mu} . \varphi \in \Phi_{r}, \delta>0, \delta^{\prime}>0$ be fixed. Choose a piecewise linear path $\dot{\varphi} \in \Phi_{x, r}$ such that

$$
\sup _{u \leq t \leq \tau}|\hat{r}(t)-\dot{\varphi}(t)|<\frac{\delta}{2} \text { and }\left|\mathcal{L}_{\tau}(\varphi)-\mathcal{L}_{\tau}(\dot{\varphi})\right|<\frac{\delta^{\prime}}{2}
$$

Then

$$
\begin{aligned}
& \frac{1}{N} \log P\left\{\sup _{t=0 \ldots,[N \tau]}\left|\frac{S_{t}([x N])}{N}-\varphi\left(\frac{t}{N}\right)\right|<\delta\right\} \\
\geq & \frac{1}{.} \log P\left\{\sup _{t=0 \ldots \ldots[N \tau]}\left|\frac{S_{t}([x N])}{N}-\dot{\varphi}\left(\frac{t}{N}\right)\right|<\frac{\delta}{2}\right\}
\end{aligned}
$$

and so to prove (ii) one should prove (ii)'.

Consider the upper bound. From compactness of the set $\Phi_{x, \tau}$ it follows that for any : $>0 . \delta>0, \delta>\sigma>0$ one can find finite number of paths

$$
\hat{r}_{1} \ldots, \hat{\gamma}_{k} \in \Phi_{j: \tau} \backslash \Phi_{r, \tau}(s)
$$

such that

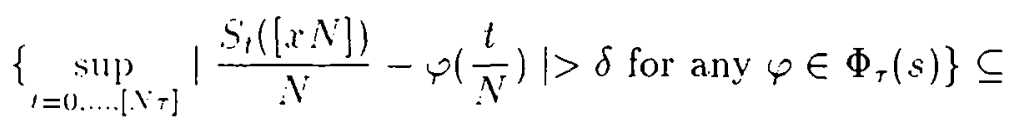

$$
\begin{aligned}
& \subseteq \bigcup_{j=1}^{k}\left\{\sup _{t=0 \ldots[N \tau]}\left|\frac{S_{t}([x N])}{N}\right|<\sigma\right\}
\end{aligned}
$$


Moreover. as for cach $\varphi \in \Phi_{r . r}$ and any $\epsilon>0$ one can find a piecewise linear path $\dot{\varphi} \in \Phi_{x . r}$ such that

$$
\operatorname{silj}_{0 \leq 1 \leq \tau}|\dot{\gamma}(t)-\dot{\varphi}(t)|<\frac{\delta}{2} \text { and }\left|\mathcal{L}_{\tau}(\varphi)-\mathcal{L}_{\tau}(\dot{\varphi})\right|<\frac{\delta^{\prime}}{2}
$$

then the paths $\gamma_{1}, \ldots, \varphi_{k} \in \Phi_{x, \tau} \backslash \Phi_{x, \tau}(s)$ can be chosen to be piecewise linear.

In other work for any $s>0, \delta>0, \delta^{\prime}>0$ one can find a finite number of piecewise linear paths $\left.\varphi_{1}, \ldots,\right\rangle_{k} \in \Phi_{x, \tau} \backslash \Phi_{x . r}(s)$ such that

$$
\begin{aligned}
& P\left\{\operatorname{silj}_{t=0 \ldots[(N)]}\left|\frac{S,([r(0) N])}{N}-\varphi\left(\frac{t}{N}\right)\right|>\delta \text { for any } r \in \Phi_{r}(s)\right\} \leq \\
& \leq \sum_{i=1}^{k} P\left\{\sup _{t=0 \ldots \ldots\left[N_{\tau}\right]}\left|\frac{S_{t}\left(\left[N_{\hat{r}_{j}}(0)\right]\right)}{N}\right|<\sigma\right\}
\end{aligned}
$$

So, (iii) follow's from (iii) . Lemma 3.1 .2 is thus proved.

Theorem 3.1.1 is proved.

\subsection{One-dimensional random walk.}

Consider a random walk in $Z_{+}^{1}$ with transition probabilities $p_{i j}, i . j \in Z_{+}^{1}$ such that

$$
p_{i, l}=m_{,-,} \text {for all } i>0, j \in Z_{+}^{1}
$$

$$
p_{i, j}=0 \text { if either }-1>j-i \text { or } j-i>d \text { for some finite } d \geq 1
$$

Note that there are only two constants $L_{\theta}=L(v, \emptyset)$ and $L(v)=L(v:\{1\})$ to be determined. The second one $L(v)$ concides with the comesponding constant for 1 he homogeneons random walk on $Z$ with transition probabilities $p_{j-i}$ and is defind below. The first one, $L_{\emptyset}$. is zero for recurrent random walks. i.e. When the mean drift $M=\sum_{i} i p_{i} \leq 0$.

Hore interwing is the case when the random walk is transient (i.e. when the mand difi $\left.H=\sum_{i} i p_{i}>0\right)$. In this case $L_{\theta}$ undergoes a kind of phase: 
transition with respect to change of parameters. We study this case in the rest of this section.

Assume the initial random walk defined on the probability space $(\Omega, \Sigma, P)$. In the sequel we use the following family of random walks $S_{t}, t \in Z_{+}, \alpha \in R$. with transition probabilities

$$
p_{i j}^{\alpha}=\frac{p_{i j} e^{\alpha(j-i)}}{\sum_{j} p_{i j} e^{\alpha(j-i)}}, p_{i j}^{0}=p_{i j}
$$

We can define these random wallis on the space $(\Omega, \Sigma)$ and shall denote the corresponding distributions and expectations by $P_{o}, E_{\alpha}$ and the random walk itself by $S_{1}^{\prime r}$. Let

$$
H_{i}(\alpha)=\log \sum_{j} p_{i j} e^{\alpha(j-i)}
$$

Then $H_{i}(\alpha)=H_{1}(\alpha)$ for all $i>0$. Note that

$$
M_{i}(\alpha)=\frac{d}{d \alpha} H_{i}(\alpha)
$$

is the mean drift for $S_{f}^{\circ}$ from the point $i$, and

$$
D_{i}(\alpha)=\frac{d^{2}}{d \alpha^{2}} H_{i}(\alpha)
$$

is the variance.

Now we can define the function $L(\cdot)$. It is the Legendre transform of the function $I_{1}$.

The graphs of $H_{1}(\alpha)$ and $H_{0}(\alpha)$ can either intersect each other on the negative $\alpha$-axis (case 2 ) or not (case 1 ) 


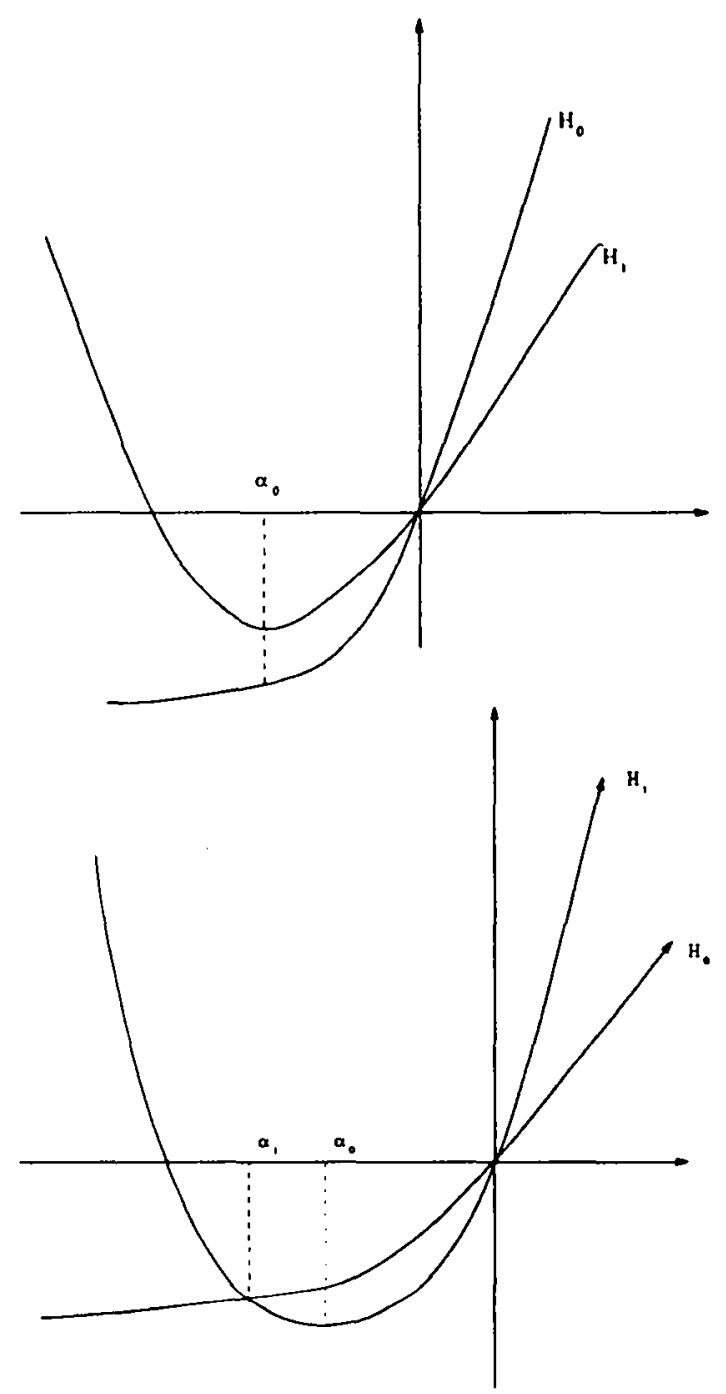

Here $a_{0}$ is such that $H_{1}\left(\alpha_{0}\right)=\min _{1} H_{1}(\alpha)$. In case 2. i.e. when

$$
H_{0}\left(\alpha_{0}\right)>H_{1}\left(\alpha_{0}\right)
$$

there exists $a_{1} \in R$ such that

$$
H_{1 \mathrm{u}}\left(\sigma_{1}\right)=H_{1}\left(\sigma_{1}\right) \text { and } \frac{d}{d \alpha^{\prime}} H_{1}\left(\alpha_{1}\right)<0
$$


Theorem 3.2.1 The large deviation principle holds for for our random wall: with the action functionals defined by the constant

$$
L_{\emptyset}= \begin{cases}H_{1}\left(\alpha_{0}\right) & \text { if } H_{0}\left(\alpha_{0}\right) \leq H_{1}\left(\alpha_{0}\right) \\ H_{1}\left(\alpha_{1}\right) & \text { if } H_{0}\left(\alpha_{0}\right)>H_{1}\left(\alpha_{0}\right)\end{cases}
$$

Proof.Choose $\delta>0$ sufficiently small and let $|x|<\frac{\delta}{2}$ Consider the random walk $S_{t}$ starting at the point $[x N]$. Denote $A_{N \delta}=\left\{\sup _{t=0 \ldots,[N \tau]} S_{t} \leq N \delta\right\}$. Then

$$
P_{0}\left(A_{N i s}\right)=E_{0} I_{A_{N S}} \exp \left\{-\alpha S_{[N \tau]}+\sum_{t=0}^{[N \tau]-1} H_{S_{t}}(\alpha)\right\}
$$

where $I_{A_{N} \delta}$ is lhe indicator of $A_{\text {Nos. }}$.

Case 2. Putting $a=\alpha_{1}$ we get the lower bound

$$
\begin{aligned}
P_{0}\left(. N_{, S}\right) & =E_{\alpha_{1}} I_{A_{N \delta}} \exp \left\{-a_{1} S_{\left[: V_{\tau}\right]}+[N \tau] H_{1}\left(\alpha_{1}\right)\right\} \\
& \geq \exp \left\{-\left|\alpha_{1}\right| \delta N+[N \tau] H_{1}\left(\alpha_{1}\right)\right\} E_{\alpha_{1}} I_{A_{N \delta}}
\end{aligned}
$$

Note that

$$
E_{a_{1}}\left[S_{t+1}-S_{i} \mid S_{t}=i\right]=M_{i}\left(a_{1}\right)<0,
$$

and thus the random walk $S_{t}^{\text {or }}$ is ergodic, and

$$
E_{\mathrm{c}, 1} I_{\text {A N }} \rightarrow 1 \text { as } N \rightarrow \infty
$$

From ( 22) and (24) we get the lower bound.

In case 2 the upper bound follows from

$P_{0}\left(A_{N \delta}\right)=E_{,, 1} I_{A, \alpha} \exp \left\{-o S_{[N \tau]}+[N \tau] H_{1}\left(\alpha_{1}\right)\right\} \leq \exp \left\{[N \tau] H_{1}\left(\alpha_{1}\right)+\left|\alpha_{1}\right| \delta N\right\}$

Case 1.

The upper bound is again trivial 


$$
\begin{aligned}
P_{u(1}\left(A_{N i}\right) & =E_{\alpha_{1}} I_{A_{N \delta}} \exp \left\{-\alpha_{0} S_{[N \tau]}+\sum_{t=0}^{[N \tau]-1} H_{S(t)}\left(\alpha_{0}\right)\right\} \\
& \leq \exp \left\{\left[N^{\prime} \tau\right] H_{1}\left(\alpha_{0}\right)+\left|\alpha_{0}\right| \delta N\right\}
\end{aligned}
$$

To get the lower bound consider the event

$$
B_{N S}=A_{N \delta} \bigcap\left\{S_{t} \neq 0 \text { for } t=0, \ldots,[N \tau]\right\}
$$

Then

$$
\begin{aligned}
& P_{0}\left(A_{1 ; j)}\right) \geq P_{0}\left(B_{, j \delta}\right)=E_{\alpha_{1}} I_{B_{N \delta}} \exp \left\{-\alpha_{0} S_{[N \tau]}+\sum_{t=0}^{\left[N_{\tau}\right]-1} H_{S_{t}}\left(\sigma_{0}\right)\right\}
\end{aligned}
$$

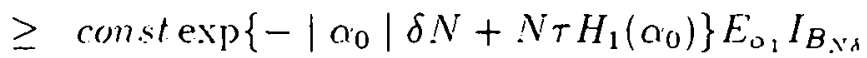

But dur to

$$
I_{i_{0}}\left\{S_{t+1}-s_{t} \mid S_{t}=i\right\}=M_{i}\left(\alpha_{0}\right)=0 . i>0 .
$$

it is easy to show that for some constant $\gamma>0$

$$
E_{\nu_{0}} I_{B, i s} \geq \gamma \exp \left\{-\dot{\delta}^{\prime} N\right\}
$$

Remark 1 The surface $\mathcal{R}=\left\{H_{1}\left(a_{0}\right)=H_{0}\left(a_{0}\right)\right\}$ is called a Reynolds surfact separating the two phases. The domain of parameters. where $L_{0}$ dors not depend on the parameters $p_{0 j}$, defines phase 1. Phase 2 is when $L_{0}$ depends on the paramelers poj. Lo depends continuously on the parameters but in general it is not differentiable at the points of $\mathcal{R}$.

\subsection{Random walk in $Z_{+}^{1} \times Z^{\mu}$}

Consider a random walk $S_{f}(i . x)$ in $Z_{+}^{1} \times Z^{\mu}$ starting at the point $(i, x)$ and having the following transition probabilities $p((i, x) \rightarrow(j, y))=p_{i, j}(x, y)$. $i, j \in Z_{+}^{1} . x . \eta \in Z^{\prime \prime}$. We shall assume that

$t_{1}$ :

(i) (homudediuess of jumps) 
$p_{i, j}(x, y)=0$. if either $j-i<-1$, or $\max \{|i-j| .|x-y|\}>d$.

for some fixed $d>0$.

(ii) (homogeneity)

$$
\begin{gathered}
p_{i, y}(x, y)=p_{1, j-i+1}(y-x) \text { for any } i>0, j \in Z_{+}^{1}, x, y \in Z^{\mu} . \\
p_{0, j}(x, y)=p_{0, j}(y-x) \text { for any } j \in Z_{+}^{1}, x, y \in Z^{\mu}
\end{gathered}
$$

(iii) the induced Markov chain with the set of states $Z_{+}^{1}$ and transition probabilities

$$
p_{i, j}=\sum_{y \in Z^{\prime \prime}} p_{i, j}(y), i, j \in Z_{+}^{1}
$$

is irreducible and aperiodic.

Consider

$$
H_{i}(\alpha, \beta)=\log \left\{\sum_{y, j} p_{i, j}(y) \exp \{\alpha(j-i)+\beta y\}\right\}
$$

$i \in Z_{+}^{1}, \alpha \in R, \beta \in R^{\mu}$.

Due to the homogeneity assumption

$$
H_{i}(\alpha, \beta)=H_{1}(\alpha, \beta)
$$

for any $i>0$. Denote by $\partial_{\alpha}, \partial_{\beta}$ the partial derivatives with respect to 0 and $\beta$ correspondingly. Here $\partial_{\beta}=\left(\partial_{\beta^{1}}, \ldots, \partial_{\beta^{\mu}}\right)$. We shall assume that jacolians of $H_{0}$ and $H_{1}$ are nowere zero:

for any $\alpha .3$

$$
\operatorname{det}\left(\begin{array}{cc}
\frac{\partial^{2}}{(\dot{\partial})^{2}} H_{0}(\alpha, \beta) & \frac{\partial^{2}}{\partial \alpha \lambda^{2} \beta} H_{0}(\alpha, \beta) \\
\frac{\partial^{2}}{\partial \alpha \partial \beta} H_{0}(\alpha, \beta) & \frac{\partial^{2}}{(\partial \beta)^{2}} H_{0}(\alpha, \beta)
\end{array}\right) \neq 0
$$

and

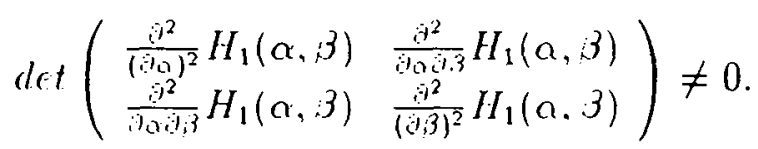

Let us consider the equations

$$
\partial_{\Omega} H_{1}(\alpha, \beta)=0
$$




$$
H_{0}(\alpha, \beta)=H_{1}(\alpha, \beta)
$$

One can easily show that for any $\beta \in R^{\prime \prime}$ there exists a unicpue $\alpha_{0}(\beta)$ for which ( 2i) holds. Let us note that for any $\beta$ for which

$$
H_{1}\left(\alpha_{0}(\beta) . \beta\right)<H_{0}\left(\alpha_{0}(\beta), \beta\right)
$$

there exists the micue $a_{1}(\beta)$ for which $(28)$ holds and

$$
\partial_{\dot{\alpha}} H_{1}(\alpha, \beta)<0
$$

Consicler he following function

$$
n(\beta)= \begin{cases}a_{0}(\beta) & \text { if } H_{1}\left(\alpha_{0}(\beta), \beta\right) \geq H_{0}\left(\alpha_{0}(\beta), \beta\right) \\ \alpha_{1}(\beta) & \text { if } H_{1}\left(\alpha_{0}(\beta), \beta\right)<H_{0}\left(\alpha_{0}(\beta), \beta\right)\end{cases}
$$

Lemma 3.3.1 The function $\mathcal{H}(\beta)=H_{1}(\alpha(\beta), \beta)$ is conver and it has continuous first derivatives.

Proof. Consider the sets

$$
\begin{aligned}
& \mathcal{C}_{0}=\left\{\beta \in R^{\mu}: H_{0}\left(\alpha_{0}(\beta), \beta\right) \leq H_{1}\left(\alpha_{0}(\beta), \beta\right)\right\} \\
& \mathcal{C}_{1}=\left\{\beta \in R^{\mu}: H_{0}\left(\alpha_{0}(\beta), \beta\right)>H_{1}\left(\alpha_{0}(\beta), \beta\right)\right\}
\end{aligned}
$$

It is rasy to see that the function $H_{1}\left(\alpha_{0}(\beta), \beta\right)$ is convex and it has a continuous first derivatives. Moreover one can easily show that the function $H_{1}\left(\alpha_{1}(3)\right.$. 3) defined on the set $\mathcal{C}_{1}$ is also convex and it also has continuous first clerivatives inside of $\mathcal{C}_{1}$.

So to prove the lemma it is sufficient to show that for any $\beta$ for which

$$
\left.H_{0}\left(\alpha_{0}(\beta), ; \beta\right)=H_{1}\left(\alpha_{0}(\beta), \beta\right)\right\}
$$

the following equalities hold

$$
\begin{aligned}
& I_{1}\left(\sigma_{0}(3) .3\right)=\lim _{\beta^{\prime} \rightarrow \beta, \beta^{\prime} \in \mathcal{C}_{1}} H_{1}\left(\alpha_{1}\left(\beta^{\prime}\right), \beta^{\prime}\right) \\
& \partial_{3} I_{1}\left(\sigma_{0}(\beta), \beta\right)=\lim _{\beta, 3,3^{\prime}, \beta^{\prime} \in C_{1}} \partial_{3^{\prime}} H_{1}\left(\alpha_{1}\left(\beta^{\prime}\right), \beta^{\prime}\right)=\partial_{3} H_{1}\left(\alpha_{1}(\beta), \beta\right)
\end{aligned}
$$


The first one is obvious. The second one can easily shown by a simple calculation.

Consider the Legendre transforms of the functions $I I_{1}(\cdot, \cdot)$ and $\mathcal{H}(\cdot)$

$$
\begin{gathered}
I_{1}\left(v^{0} \cdot \bar{v}\right)=\sup _{\alpha \in R, \beta \in R^{\mu}}\left\{a v^{0}+3 \bar{v}-H_{1}(\alpha, \beta)\right\} \\
L_{0}(\bar{v})=\sup _{\beta \in R^{\mu}}\{3 \bar{v}-\mathcal{H}(3)\}
\end{gathered}
$$

Let

$$
l .\left(\left(x^{0} \cdot \bar{x}\right) \cdot\left(r^{0} \cdot \bar{c}\right)\right)= \begin{cases}L_{1}\left(r^{0} \cdot \bar{v}\right) & \text { if } x^{0}=0 \\ L_{0}(\bar{c}) & \text { otherwise }\end{cases}
$$

For each continuous path $\hat{\tau}:[0 . \tau] \rightarrow R_{+}^{1} \times R^{\mu}$ we define

$$
\mathcal{L}-(\hat{r})= \begin{cases}\int_{0} L(\dot{\hat{r}}(t) \cdot \hat{r}(t)) d t . & \text { if } \dot{\varphi} \text { is absolutely continuous, } \\ +\infty & \text { otherwise. }\end{cases}
$$

Theorem 3.3.1 The random walk $S_{t}$ satisfies the large deviation principle with the action functionals $\mathcal{L}_{T}$.

Proof. Dne to the theorem 3.1 .1 we should show that for any $\tau>0$ the following conctusions hold.

1. The functional $\mathcal{L}_{\tau}$ is lower semicontinuous.

2. For any $x \in R_{+}^{1} \times R^{u}, y \in \Phi_{x \cdot \tau}$ and for any $\epsilon>0$ there exists a pieceswise linear path $\dot{\varphi} \in \Phi_{x, r}$ such that

$$
\sup _{1 u \leq i \leq-}|\gamma(1)-\dot{\gamma}(t)|<\epsilon \text { and }\left|\mathcal{L}_{\tau}(\hat{\gamma})-\mathcal{L}_{\tau}(\dot{\varphi})\right|<\epsilon
$$

3. The lower large deviation bound ( 16) and the upper large deviation bound $(1 i)$ hold for any linear path $p$. 
The proof of the two first conclusions is quite standard.Let us prove the lower large deviation bound (16) and upper large deviation bound (17).

Lemma 3.3.2 lit $\varphi:[0 . \tau] \rightarrow R_{+}^{1} \times R^{\mu}, \varphi(t)=\left(\varphi^{0}(t), \bar{\varphi}(t)\right)$ be a limear path such that for an! $0<t<\tau \quad \varphi^{0}(t) \neq 0$ and $\dot{\varphi}(t)=v$ ?

Then the lourer large deviation bound (16) and the upper large deviation bound ( 17) hold with

$$
\mathcal{L}_{\tau}(\vartheta)=\tau L_{1}(v)
$$

This lemma casily follows from the large deviation principle for homogeneous random wallis in $Z^{n+1}$ (see [9])

Lemma 3.3.3 l.cl $\hat{\varphi}:[0 . \tau] \rightarrow R_{+}^{1} \times R^{\mu} \cdot \nu(t)=\left(\hat{\varphi}^{0}(t) . \bar{\varphi}(t)\right)$ be a lincar path such that for any $t \in[0 . \tau] \quad \hat{P}^{0}(t)=0$ and $\dot{\bar{\varphi}}(t)=\bar{v}$.

Then the louer large deviation bound (16) and the upper large deviation bound ( 17) hold with

$$
\mathcal{L}_{\tau}(\varphi)=\tau L_{0}(\bar{v})
$$

where $\bar{\imath}=\dot{\bar{s}}(1)$

Proof. let $\bar{x}=\dot{\bar{\varphi}}(1) . \varphi(0)=0 . x \in R_{+}^{1} \times R^{\mu} .|x|<\frac{s}{2}$. Consider the random walk $S_{t}=\left(S_{t} . \bar{S}_{t}\right)$ starting at the point $[x . \%]$. Denote

$$
\begin{gathered}
A_{N S}^{0}=\left\{\sup _{t=0 \ldots N_{T}}\left|S_{t}^{0}\right|<N \delta\right\} . \\
. t_{N i}^{1}=\left\{\sup _{t=0 \ldots \ldots N_{T}}\left|\bar{S}_{t}-\bar{\tau} t\right|<N \delta\right\} .
\end{gathered}
$$

and

$$
A_{N}=\left\{\sup _{t=0 \ldots \alpha_{\tau}}\left|s_{t}-\varphi\left(\frac{t}{N}\right)\right|<N \delta\right\}=A_{N S}^{0} \cap A_{N i}^{1}
$$

Consicler a family of random walks in $Z_{+}^{1} \times Z^{\mu}$ with transition probabilities

$$
\begin{aligned}
& p^{0 \cdot 3}((i \cdot x) \cdot(j \cdot y))=p_{i, j}^{a, 3}(x \cdot y)= \\
& p_{i, j}(x, y) \exp \left\{a(j-i)+\beta(y-x)-H_{i}(\alpha . j)\right\}= \\
& =\frac{\left.p_{i . j}(x \cdot y) \exp \right)\{a(j-i)+3(y-x)\}}{\sum_{: !} p_{i . j}(x \cdot y) \exp \{a(j-i)+3(\bar{y}-\bar{x})\}} \text {. }
\end{aligned}
$$


Then

$$
\eta_{i, j}^{u, u}(x, y)=p_{i, j}(x, y), \quad i, j \in Z_{+}^{1}, x, y \in Z^{\mu}
$$

Nor shall demote the corresponding distributions and expectations by $P_{a, 3,} E_{n, 3}$. One can easily show that for any $a \in R^{1}, \beta \in R^{\prime \prime}$

$$
\begin{aligned}
P\left\{A_{N \delta \delta}\right\} & =P_{0.0}\left\{A_{N \delta}\right\}= \\
& =E_{0,3}\left(I_{A_{N^{\prime} \delta}} \exp \left\{-\alpha S_{[N \tau]}^{0}-\beta \bar{S}_{[N \tau]}+\sum_{t=0}^{[N \tau]-1} H_{S_{t}^{0}}(\alpha, \beta)\right\}\right)
\end{aligned}
$$

where $I_{A, s}$ is an indicator of $A_{N s}$.

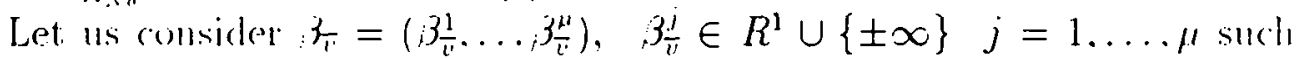
that

$$
\begin{aligned}
L_{0}(\bar{v}) & =\inf _{\beta}\left\{\bar{v} \beta-H_{1}(\alpha(\beta), \beta)\right\}= \\
& =\bar{v} \beta_{\bar{v}}-H_{1}\left(\alpha\left(\beta_{\bar{v}}\right), \beta_{\bar{v}}\right)
\end{aligned}
$$

Let us first prove the lower large deviation bound and upper large doriation bound for the case when

$$
\left|.3 \frac{j}{l^{\prime}}\right|<\infty \text { for any } j=1, \ldots, \mu
$$

Then from the clefinition of the function $\alpha(\beta)$ it follows that

$$
\left|\alpha\left(\beta_{\bar{v}}\right)\right|<\infty
$$

From $(30)$ for $, \beta=\beta_{-}, a=a\left(\beta z^{\prime}\right)$ we get

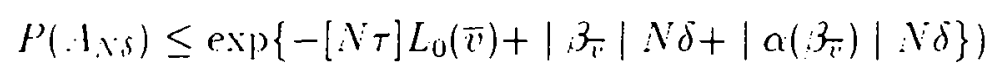

So for the case when (32) holds the upper large deviation bound is proved.

Let us prow now the lower large deviation bound for the case when (32) holds. We ronsider here two cases:

(ascl.

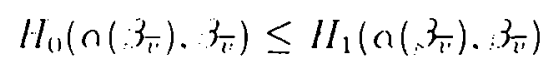

Casc 2.

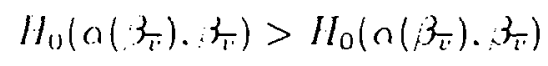


Let ( 3-4) be satisfied. Then by definition

$$
\alpha\left(\beta z_{v}\right)=\alpha_{0}\left(\beta_{\bar{v}}\right)
$$

and consequently for $a_{\bar{v}}=\alpha\left(\beta_{\bar{v}}\right)$

$$
\begin{aligned}
& \left.\partial_{1} H_{1}\left(\alpha_{\bar{v}}\right), \beta \beta_{\bar{v}}\right)=0 \\
& \left.\partial_{3} H_{1}\left(\alpha_{\bar{v}}\right), \beta_{\bar{v}}\right)=\bar{v}
\end{aligned}
$$

From ( 36$)$ it follows that

$$
\begin{aligned}
& E_{0,(3, i) i t}\left(S_{t+1}^{0}-S_{t}^{0} \mid S_{t}^{0}=i, \bar{S}_{t}\right)=0 \\
& E_{0\left(\beta_{T}\right), B_{n}}\left(\bar{S}_{t+1}-\bar{S}_{t} \mid S_{t}^{0}=i . \bar{S}_{t}\right)=\bar{r}
\end{aligned}
$$

for any $i \in \%_{+}^{1} . \quad i>0$. Consider $i_{0} \in Z_{+}^{1}, i_{0}>0$ and $y \in Z^{\mu}$ such that

$$
p_{1}\left(i_{0}, y\right)>0
$$

Let

$$
p_{\Lambda}\left(i_{0}, y\right)=q
$$

Then for $\Lambda_{i}=[\sqrt{N}]$

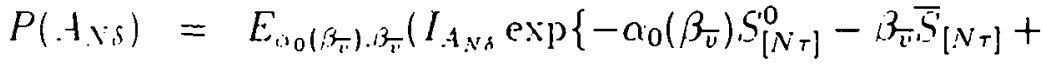

$$
\begin{aligned}
& \left.\left.+\sum_{t=0}^{\left[N_{\tau}\right]-1} H_{S_{1}^{0}}\left(\rho_{v}\left(\beta_{\bar{\tau}}\right), \beta_{\bar{v}}\right)\right\}\right) \geq \\
& \geq q^{N_{1}} \exp \left\{-[N \tau] L_{0}(\bar{v})-\left|\beta_{\bar{v}}\right| N \delta-\left|\alpha\left(\beta_{\bar{v}}\right)\right| N \delta\right\} \times \\
& \times P_{n_{0}\left(B_{T}\right) \ldots \tau}\left\{B_{N_{1}, N S} \mid S_{N_{1}}^{0}=\epsilon_{0} N_{1}, S_{N_{1}}=N_{1} y\right\}
\end{aligned}
$$

where

$$
\begin{gathered}
B x_{i} \ldots, i,=\left\{\inf _{t=v_{1} \ldots \ldots v_{\tau}}\left|S_{t}^{0}\right|>0, \sup _{t=N_{1} \ldots, N_{\tau}}\left|S_{t}^{0}\right|<N \delta,\right. \\
\left.\sup _{t=V_{1} \ldots N_{\tau}}\left|\bar{S}_{t}-\bar{v} t\right| \delta N\right\}
\end{gathered}
$$

From ( 37 ) it anity follows that there exists $c>0$ such that

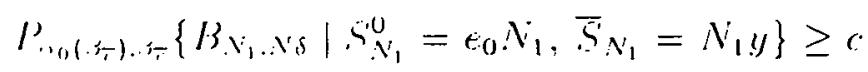


From ( 38 ) and ( 39$)$ we get for the case 1 the lower large deviation bound.

Let us consider now the case 2. For this case by definition

$$
a\left(\beta_{-}\right)=\alpha_{1}\left(\beta_{\bar{R}^{\prime}}\right)
$$

where $\alpha\left(\beta_{\overline{v^{\prime}}}\right)$ is defined by the system

$$
\left\{\begin{array}{c}
H_{0}\left(\alpha_{1}\left(\beta_{\bar{v}^{\prime}}\right), \beta_{\bar{v}^{\prime}}\right)=H_{1}\left(\alpha_{1}\left(\beta_{\bar{v}}\right), \beta_{\bar{v}}\right) \\
\partial_{\alpha} H_{1}\left(\alpha_{1}\left(\beta_{\bar{v}^{\prime}}\right), \beta_{\bar{v}^{\prime}}\right)<0 .
\end{array}\right.
$$

Therefore from ( 30$)$ for $n=n_{1}\left(3_{z^{2}}\right), 3=\beta_{z^{\prime}}$ one can easily get

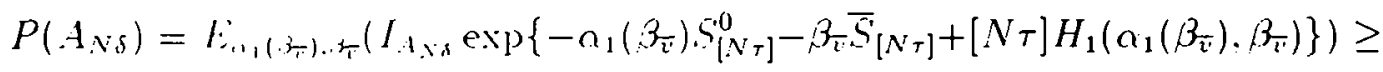

$$
\begin{aligned}
& \geq \exp \left\{-N_{T} L_{0}(\bar{\imath})-N \delta\left(\left|\alpha_{1}\left(\beta_{\bar{v}}\right)\right|+\left|\beta_{\bar{v}^{\prime}}\right|\right)\right\} \times \\
& \times P_{0,1 ;}\left(z_{1}\right) \cdot 3_{\pi}\left(A_{N \delta}\right)
\end{aligned}
$$

Let us prove now the following lemma.

Lemma 3.3.4 l.ft (.35) holds then

$$
\left.P_{0,1}(.)_{T}\right) \cdot 3_{2}\left(A_{N \delta}\right) \rightarrow 1 \text { as } N \rightarrow \infty
$$

Proof Comsicle for any $n, \beta$ the induced Markov chain with the set of states $Z_{+}$and transilion probabilities

$$
p_{i, j}^{0,3}=\sum_{y} p_{i, j}^{\alpha \beta}(y)
$$

Let ( 35$)$ he satisfied. Then for $a=\alpha_{1}\left(\beta_{\bar{v}}\right), \beta=\beta_{\overline{v^{*}}}$ the induced Markos: chain is ergorlic. ('onsider for any $a .3$ for which the induced Markov chain is ergodic the stationary probabilities of this chain

$$
\pi_{i}^{0.3}, j \in Z_{+}^{1}
$$

and consider the rector

$$
V_{0}(0 . ; 3)=\pi_{0}^{0.3} \sum_{y . j} y p_{0 . j}^{0,3}(y)+\left(1-\pi_{0}^{0, \beta}\right) \sum_{y, j} y p_{1 . j}^{0, \beta}(y)
$$


From the theorem 4.1.1 it easily follows that to prove the lemma 3.3.4 it is sufficient to show that

$$
\bar{v}=V_{0}\left(\alpha_{1}\left(\beta_{\bar{v}}\right), \beta_{\bar{v}}\right)
$$

Let us calculate $V_{0}(\alpha, \beta)$. For this let us first note that for any $\alpha, \beta$

$$
\sum_{j, y} y p_{i, j}^{\alpha, \beta}(y)=\partial_{\alpha} H_{i}(\alpha, \beta)
$$

Moreover using the method of generating functions one can easily show that for each $\alpha, 3$ for which the induced Markov chain is ergodic

$$
\pi_{0}^{u \cdot \beta}=\frac{\partial_{\alpha} H_{2}(\alpha, \beta)}{\partial_{0,0} H_{0}(\alpha, \beta)-\partial_{\alpha} H_{1}(\alpha, \beta)}
$$

From ( 40) and ( 41$)$ we get

$$
V_{0}(\alpha, \beta)=\frac{\partial_{\alpha}\left(H_{0}(\alpha, \beta)-H_{1}(\alpha, \beta)\right.}{\partial_{0} H_{1}(\alpha, \beta) \partial_{3} H_{0}(\alpha, \beta)-\partial_{\alpha} H_{0}(\alpha, \beta) \partial_{3} H_{1}(\alpha, \beta)}
$$

By a simple calculation one can easily show that for those 3 for which

$$
\mathcal{H}_{0}(\beta)=H_{0}\left(\alpha_{1}(\beta), \beta\right)=H_{0}\left(\alpha_{1}(\beta), \beta\right)
$$

the following equality holds

$$
\frac{d}{d ;} \mathcal{H}_{u}(\beta)=\frac{\partial_{c}\left(H_{0}(\alpha, \beta)-H_{1}(\alpha, \beta)\right)}{\partial_{v} H_{1}(\alpha, \beta) \partial_{3 \beta} H_{0}(\alpha, \beta)-\partial_{\alpha} H_{0}(\alpha, \beta) \partial_{3} H_{1}(\alpha, \beta)}
$$

But for $\beta=\beta_{\text {r }}$ from the definition of $\beta_{2}$ it follows that

$$
\frac{d}{d i} \mathcal{H}_{0}\left(\beta_{\bar{v}}\right)=\bar{v}
$$

Consequently from (12) and (43) we get

$$
I_{0}\left(\Omega_{1}\left(\beta_{\bar{v}^{\prime}}\right) \cdot\left(\beta_{\bar{v}^{\prime}}\right)=\bar{v}\right.
$$

Lemma 3.3 .4 is proved.

We have proved the lower large deviation bound and upper large deviation bound for the ase when for any $j=1 \ldots . . \mu$.

$$
\left|3 \frac{j}{1}\right|<\infty
$$


To consider the case when for some $1 \leq j \leq \mu$

$$
\left|\beta_{\bar{v}}^{j}\right|=\infty
$$

let us note that for any $j=1, \ldots, \mu$, for which $\beta \frac{!}{l^{\prime}}=\infty$, if the trajertory belongs to the event $A_{N \delta}$ then

$$
S_{t}^{j}=t v^{j} \text { for any } t=0, \ldots,\left[\Lambda_{\tau}\right]
$$

Considering now conditional distributions under the condition

$$
S_{t}=t c^{j} \text { for any } t=0, \ldots,[N \tau] \text { for any } j \text { for which }\left|, \beta \frac{j}{v^{\prime}}\right|=\infty .
$$

and using for these conditional distributions the same arguments as in previous case one can easily get the lower and upper large deviation bounds.

The lemma 3.3.3 is proved.

\subsection{Random walk in $Z_{+}^{\mu+1}$ with a discontinuity on a hyperplane}

Let us consicler the Markov chain with the set of states $Z^{\mu+1}$ ( denote its state at time $t$ by $S_{t}(i, x)$ if the Markov chain starts from the point $\left.(i, x)\right)$ and having the following transition probabilities

$$
p((i, x) \rightarrow(j, y))=p((i, x),(j, y)), i, j \in Z^{1}, x, y \in Z^{\mu} .
$$

We shall assume that

(i) (boundedness of jumps)

$p_{i, j}(x, y)=0$. if either $\max \{|i-j|,|x-y|\}>d$. or $(j-i) \operatorname{sign}(i)<-1$ for some $d>0$ :

(ii) (homogeneity) for all $(i, x),(j, y),(k, z)$ such that $\operatorname{sign}(i)=\operatorname{sign}(i)$ we have

$$
p((i, x),(j, y))=p((i+k, x+z),(j+k, y+z))
$$

Put

$$
p((i, x),(j \cdot y))=p_{i j}(y-x)
$$


(iii) the indued Markov chain having the set of states $Z^{1}$ and transition probabilitios:

$$
p_{i, j}=\sum_{y} p_{i, j}(y)
$$

is irreducible and aperiodic.

Define the following functions

$$
H_{+}(\alpha . \beta)=\log \left(\sum_{j, y} p_{i, j}(y) \exp \{\alpha(j-i)+\beta y\}\right)
$$

where $i>0 . n \in R^{1} .3=\left(\beta^{1} \ldots, \beta^{\mu}\right) \in R^{\mu}$, and for $y=\left(y^{1}, \ldots, y^{\mu}\right)$ we put.

$$
\begin{gathered}
\beta y=\sum_{k=1}^{\mu} \beta^{k} y^{k} \\
I_{-}(\gamma, \beta)=\log \left(\sum_{j, y} p_{i, j}(y) \exp \{-\gamma(j-i)+\beta y\}\right)
\end{gathered}
$$

where $i<0, i \in R$. and

$$
H_{0}(\text { o.3.; })=\log \left(\sum_{y, l \geq 0} p_{0, j}(y) \exp \{\alpha j+\beta y\}+\sum_{y, j<0} p_{0, j}(y) \exp \{-\gamma j+\beta y\}\right)
$$

Assume that the jacobians of $H_{+}, H_{0}$ and $H_{-}$are nonwhere zero.

Let us consiclor the following equations

$$
\begin{aligned}
& \partial_{\alpha} H_{+}(\alpha, \beta)=0 \\
& \partial_{\gamma} H_{-}(\gamma, \beta)=0
\end{aligned}
$$

It is easy 10 see that for each $\beta \in R^{\mu}$ there exists a unique solution $\left(\alpha_{0}(3) .3\right)$ of the equation $(44)$ and a unique solution $\left(\gamma_{0}(\beta), \beta\right)$ of the equation $(-15)$.

Lemma 3.4.1 l.r'

$$
\max \left\{H_{+}\left(\alpha_{0}(\beta) . ; \beta\right) . H_{-}\left(\alpha_{0}(\beta), \beta\right)\right\}<H_{0}\left(\alpha_{0}(\beta), \beta, \gamma_{0}(\beta)\right)
$$

Then one of lle following conclusions holds 
1. either.

$$
I_{+}\left(\operatorname{ou}_{0}(3) .3\right)<H_{-}\left(\gamma_{0}(3) .3\right)<H_{0}\left(\cap_{0}(3) .3, \gamma_{0}(3)\right)
$$

and three frists $n_{1}(3)$ such that

$$
H_{+}\left(\alpha_{1}(3) . .3\right)<H_{-}(\gamma 0(\beta), \beta)=H_{0}\left(\alpha_{1}(\beta), \beta . \gamma_{0}(3)\right)
$$

and

$$
\partial_{n} H_{+}\left(\alpha_{1}(\beta) . \beta\right)<0
$$

2. or

$$
I_{-}(30(3) .3)<H_{+}\left(o_{0}(3), 3\right)<H_{0}\left(a_{0}(3), \beta, \gamma_{0}(3)\right)
$$

and there atists in (3) such that

$$
I_{-}\left(\beta_{11}(\beta), \beta\right)<H_{+}\left(\alpha_{0}(\beta), \beta\right)=H_{0}\left(\alpha_{0}(\beta), \beta, \gamma_{1}(\beta)\right)
$$

and

$$
\partial_{-} H_{-}\left(\gamma_{1}(\beta), \beta\right)<0
$$

3. or the re frist $\mathrm{o}_{2}(3)$ and $i_{2}(3)$ such that

$$
I_{+}\left(\alpha_{2}(3), \beta\right)=H_{-}\left(\gamma_{2}(\beta), \beta\right)=H_{0}\left(\alpha_{2}(\beta), \beta, \gamma_{2}(\beta)\right)
$$

aind

$$
\begin{aligned}
& \partial_{1} H_{+}\left(\alpha_{2}(\beta), \beta\right)<0 \\
& \partial_{\alpha_{1}} H_{-}\left(\gamma_{2}(\beta), \beta\right)<0
\end{aligned}
$$

\section{Proof of the lemma 3.4.1}

Let us comsiclen the case when

$$
H_{+}\left(\sigma_{0}(3) .3\right) \leq H_{-}\left(\gamma_{0}(\beta) .3\right)<H_{0}\left(\alpha_{0}(\beta) . ; 3 \gamma_{10}(\beta)\right)
$$

The case when

$$
I_{-}\left(\beta_{0}(3) .3\right)<H_{+}\left(\sigma_{0}(3), 3\right)<H_{0}\left(\alpha_{0}(\beta) .3 . \beta_{0}(3)\right)
$$

can be comsidered similarly. 
Note that $H_{0}(\alpha, \beta, \gamma)$ is monotone increasing in $\alpha$ for all $\beta, \gamma$, and the function $H_{+}(0.3)$ is convex in a for all $\beta$. From this it follows that either there exists $\alpha_{1}(\beta)<\alpha_{0}(\beta)$ such that

$$
H_{+}\left(\alpha_{1}(\beta), \beta\right)<H_{-}\left(\gamma_{0}(\beta), \beta\right)=H_{0}\left(\alpha_{1}(\beta), \beta, \gamma_{0}(\beta)\right)
$$

and

$$
\partial_{0} H_{+}\left(\alpha_{1}(\beta), \beta\right)<0
$$

or there exists $\delta(\beta)<\alpha_{0}(\beta)$ such that

$$
H_{+}(i(\beta) .3)=H_{-}(-i 0(\beta), \beta) \leq H_{0}(\alpha(3), 3.70(3))
$$

Let us show that from $(46)$ it follows that there exist $\alpha_{2}(\beta)$ and $\gamma_{2}(;)$ such that

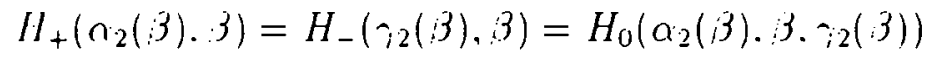

and

$$
\begin{aligned}
& \partial_{0} H_{+}\left(\alpha_{2}(\beta), \beta\right)<0 \\
& \partial_{\gamma_{i}} H_{-}\left(\gamma_{2}(\beta), \beta\right)<0
\end{aligned}
$$

For this let us consider the equation

$$
H_{+}(\cap, \beta)=H_{-}(\gamma, \beta)
$$

If $(46)$ holds then one can easily show that for each $;<90(3)$ there exists the unique $n(3, \gamma)<a(\beta)$ such that $(\alpha(\beta, \gamma), \beta, \gamma)$ is a solution of $(48)$, and moreover $O(\xi, \gamma)$ is monotone increasing in $\gamma$, and

$$
a(3 . i) \rightarrow-\infty \text { as } i \rightarrow-\infty
$$

Note that

$$
H_{-}(;, 3)=H_{+}(\alpha(3, \gamma), 3) \rightarrow+\infty
$$

as $? \rightarrow-\infty$ and the function $H_{0}(\alpha(\beta . \gamma), \beta . \gamma)$ is monotone decreasing as $i \rightarrow-x$. Then there exists the unique $\gamma_{2}(3)<\gamma_{0}(3)$ such that

$$
I_{+}\left(0\left(.3 .7_{2}(3)\right) .3\right)=H_{-}(. ; 2(3), 3)=H_{0}\left(a\left(3.7_{2}(3)\right), 3.72(3)\right)
$$


Since $\gamma_{2}(\beta)<\gamma_{10}(\beta)$ and $\alpha\left(\beta . \gamma_{2}(\beta)\right) \leq \alpha(\beta)<\alpha_{0}(\beta)$ then

$$
\begin{gathered}
\partial_{w} H_{+}\left(\alpha\left(\beta, \gamma_{2}(\beta)\right), \beta\right)<0 \\
\partial_{\gamma} H_{-}\left(\gamma_{2}(\beta), \beta\right)<0
\end{gathered}
$$

So, putting $\alpha_{2}(\beta)=\alpha\left(\beta, \gamma_{2}(\beta)\right)$ we get $(47)$.

Lemma 3.4 .1 is proved.

Let us define the function $\mathcal{H}(\beta)$ by putting

$$
\mathcal{H}(: 3)=\max \left\{H_{+}\left(\alpha_{0}(\beta) \cdot \beta\right), H_{-}\left(\alpha_{0}(3) \cdot 3\right)\right\}
$$

if

$$
\max \left\{H_{+}\left(\alpha_{0}(\beta), \beta\right) . H_{-}\left(\alpha_{0}(\beta), \beta\right)\right\} \geq H_{0}\left(\alpha_{0}(\beta) . \beta . \gamma_{0}(\beta)\right)
$$

Otherwise we put

$$
\mathcal{H}(\beta)=H_{-}\left(\gamma_{0}(\beta), \beta\right)
$$

if the case 1 of the lemma 3.4.1 holds, or

$$
\mathcal{H}(\beta)=H_{+}\left(\alpha_{0}(\beta), \beta\right)
$$

if the case 2 of the lemma 3.4 .1 holds. or

$$
\mathcal{H}(\beta)=H_{-}\left(\gamma_{2}(\beta), \beta\right)=H_{+}\left(\alpha_{2}(\beta), \beta\right)
$$

if the case 3 of the lemma 3.4 .1 holds. Then the function $\mathcal{H}$ is defined for all $\beta$.

Lemma 3.4.2 The function $\mathcal{H}$ is conver and belongs to $C^{1}\left(R^{\mu}\right)$.

Proof of the lemma 3.4.2.

Introduce the following sets

$$
\begin{gathered}
\mathcal{C}_{+}=\left\{, 3 \in R^{\prime \prime}: \mathcal{H}(\beta)=H_{+}\left(\sigma_{0}(\beta), 3\right)\right\} . \\
\mathcal{C}_{-}=\left\{\beta \in R^{\prime \prime}: \mathcal{H}(\beta)=H_{-}\left(\gamma_{0}(\beta), 3\right)\right\} . \\
\mathcal{C}_{0}=\left\{; \in R^{\prime \prime}: \mathcal{H}(3)=H_{+}\left(\alpha_{2}(\beta), \beta\right)=H_{-}\left(\gamma_{2}(3), 3\right)\right\} .
\end{gathered}
$$

Insicle of each of these sets $\mathcal{H}$ is convex and smooth. 
Let $\mathcal{C}_{+} \cap \mathcal{C}_{0} \neq \emptyset$. Consider $\beta^{*} \in \mathcal{C}_{+} \cap \mathcal{C}_{0}$. It can be verified by the direct calculation that the function $\mathcal{H}$ is continuous in $\beta^{*}$ and

$$
\lim _{\beta \rightarrow i^{*}, ; \in \mathcal{C}_{+}} \Gamma \mathcal{H}(\beta)=\lim _{\beta \rightarrow \beta^{*}, 3 \in \mathcal{C}_{0}} \nabla \mathcal{H}(\beta)
$$

Then the function $\mathcal{H}$ is convex on the set $\mathcal{C}_{+} \cap \mathcal{C}_{0}$ and it has continuous first derivatives insick this set.

Similarly. one can show that $\mathcal{H}$ is convex on the set $\mathcal{C}_{-} \cap \mathcal{C}_{0}$ and also has continuous first derivatives inside this set.

Note also that $\mathcal{C}_{+} \cap \mathcal{C}_{-}=\emptyset$. Then $\mathcal{H}$ is convex on the set

$$
\mathcal{C}_{+} \cap \mathcal{C}_{0} \cap \mathcal{C}_{-}=R^{\prime \prime}
$$

and it is of the class $C^{+1}\left(R^{\prime \prime}\right)$.

Lemma 3.1 .2 is proved.

Let $I_{+}, I_{-}$and $L_{0}$ be the Legendre transforms of the functions $H_{+}, H_{-}$ and $\mathcal{H}$ correspondingl!:

$$
\begin{gathered}
L_{+}(u, v)=\sup _{\alpha, \beta}\left\{\alpha u+\beta v-H_{+}(\alpha, \beta)\right\} \\
L_{-}(u, v)=\sup _{\gamma, \beta}\left\{\gamma u+\beta v-H_{-}(\gamma, \beta)\right\} \\
L_{0}(v)=\sup _{\beta}\{\beta v-\mathcal{H}(\alpha, \beta)\}
\end{gathered}
$$

Consider the following function $L: R^{\mu+1} \times R^{\mu+1} \rightarrow R$

$$
L\left((u, v),\left(x_{0}, x\right)\right)= \begin{cases}L_{+}(u, v) & \text { if } x^{0}>0 \\ L_{-}(u, v) & \text { if } x^{0}<0 \\ L_{0}(v) & \text { if } x^{0}=0\end{cases}
$$

For each $\tau \geq 0$ let us define on the set of all continuous paths

$$
\hat{\tau}:[0 . \tau] \rightarrow R^{\mu+1}
$$

the functional $L_{\text {. }}$.

$$
\dot{\mathcal{L}}_{T}(\varphi)=\int_{i}^{\tau} L(\dot{\varphi}(t), \varphi(t)) d t
$$


if the path $\hat{i}$ is absolutely continuous, and

$$
\mathcal{L}_{r}(Y)=\infty
$$

otherwise.

Theorem 3.4.1 For the random walk Sit the large deviation principle holds with the aclion functionals $\mathcal{L}_{\text {r }}$.

\subsection{Optimal paths.}

Consider a andom walk $S_{t} t \in Z_{+}$in $Z_{+}^{\prime \prime} \times Z^{\prime \prime}$ defined in section 3.1.Assume that this random walk satisfies the large deviation principle with the action functionals $\mathcal{L}_{-}$.

For any $x, y \in R_{+}^{\prime \prime} \times R^{\prime \prime}$ let us consider the set of all continuous paths going from $x$ to $y$. lle shall denote it by $\Phi^{x \cdot y}$.

Definition 3.5.1 Consider tuo points $x, y \in R_{+}^{\prime \prime} \times R^{\mu}$.

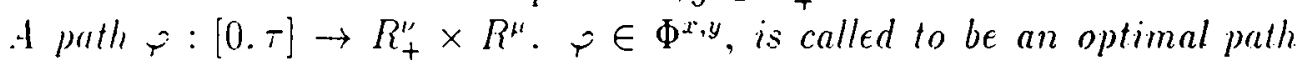
from the proinl $x$ to the point $y$ if for any $\tau^{\prime}$ and for any path $\varphi^{\prime}:\left[0, r^{\prime}\right] \rightarrow$ $R_{+}^{\prime \prime} \times R^{\prime \prime} . q^{\prime} \in \Phi^{x \cdot u}$.the following inequality holds

$$
\mathcal{L}_{\tau}\left(\hat{\tau^{\prime}}\right) \leq \dot{\mathcal{L}}_{\tau^{\prime}}\left(\hat{\varphi}^{\prime}\right)
$$

Definc

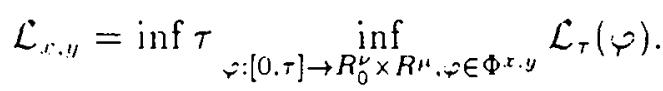

If there exists an optimal path $\hat{\gamma}:[0 . \tau] \rightarrow R_{+}^{\prime \prime} \times R^{\mu}$ from the point $x$ to the point y then

$$
\dot{L}_{x, y}=\dot{L}_{\tau}(\hat{r})
$$

Consider some properties of the optimal paths.

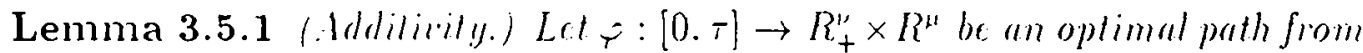
the point x to lles point y. The for any $t \in[0, \tau]$ the path $=:[0, t] \rightarrow R_{+}^{\prime} \times R^{\prime \prime}$ is optimal fiom $x$ to $z=\sigma_{-}(t)$. the path $\hat{\tau}:[t, \tau] \rightarrow R_{+}^{\prime \prime} \times R^{\mu}$ is optimal from z to $y$. and

$$
\dot{\mathcal{L}}_{r, y}=\mathcal{L}_{r, z}+\mathcal{L}_{z: y}
$$


This lemma easily follows from the integral representation of the action functional.

Definition 3.5.2 We shall say that an affine mapping

$$
G: R_{+}^{\prime \prime} \times R^{\mu} \rightarrow R_{+}^{\prime \prime} \times R^{\mu}
$$

is a proper mapping iff for any face $\Lambda$

$$
C_{i}(\Lambda)=\Lambda
$$

It is easy to see that for any proper mapping $G$ there exist $k \in R_{+}$and $b=\left(0, b^{2}\right) \in R_{+}^{\prime \prime} \times R^{\prime \prime}$ such that

$$
G(x)=k x+b .
$$

Lemma 3.5.2 (Inuariance with respect to proper mappings.) Let $\mathrm{\varphi}:[0, \tau] \rightarrow$ $R_{+}^{\nu} \times R^{\mu}$ be an optimal path from $x$ to $y$.

Then for any proper mapping $G^{\prime}(x)=k x+b$ the path $\varphi^{\prime}:[0 . k \tau] \rightarrow$ $R_{+}^{\nu} \times R^{u}$. where $\varphi^{\prime}(t)=k_{-}(k+t)+b . t \in R_{+}$, is an optimal path from the point $G(x)$ to the point $G(y)$

This lemma easily follows from the definition of the randon walk $S_{t}$. Let $p\left((x, y) \rightarrow\left(x^{\prime}, y^{\prime}\right)\right) \cdot(x, y),\left(x^{\prime}, y^{\prime}\right) \in Z_{+}^{\nu} \times Z^{\prime \prime}$ be the transition probabilities of the random walk s, Then. by clefinition, for any face.$I$ and for any $(x, y) \in$ $\Lambda \cap Z_{+}^{\nu} \times Z^{\mu}$

$$
p\left((x, y) \rightarrow\left(x^{\prime} \cdot y^{\prime}\right)\right)=p\left(\Lambda .\left(x^{\prime}-x \cdot y^{\prime}-y\right)\right),\left(x^{\prime}, y^{\prime}\right) \in Z_{+}^{\prime \prime} \times Z^{\prime \prime} .
$$

For each face 1 consider the function

$$
H_{1}^{\prime \prime \mu}(n, 3)=\sum_{x \in Z_{+}^{\prime \prime}, y \in Z^{n}} p(\Lambda,(x, y)) \exp \{0 x+3 y\}
$$

For $\nu=0$ we should consider only the face $\Lambda=\{\emptyset\}$ and then

$$
H_{\{0\}}^{0.1 \prime}(0.3)=H^{\mu}(3)
$$

Consider the legendere transform $L^{\mu}(\cdot)$ of the function $H^{\mu}(\cdot)$. 
For $1=0$ the random walk $S_{t}$ in $Z^{\prime \prime}$ is homogentous by definition and it satisfied the latge deviation principle with the action functionals $\mathcal{L}_{\tau}$ where:

$$
\mathcal{L}_{r}(\dot{r})=\int_{0}^{T} L^{\mu}(\dot{p}(t)) d t
$$

if the path $\varphi:[0 . \tau] \rightarrow R^{\prime \prime}$ is absolutely continuous and

$$
\mathcal{L}_{T}(\varphi)=\infty
$$

otherwise (sec $[9]$ ).

Theorem 3.5.1 Let $\nu=0$. and

$$
\nabla H^{\mu}(0) \neq 0 \text {. }
$$

Then for any $x \neq y \in R^{\mu}$ there exists the unique optimal path $:[0 . \tau] \rightarrow R^{\prime \prime}$ from the point $x$ to the point $y$. This optimal path is linear

$$
\varphi(t)=x+\frac{t}{\tau}(y-x)
$$

and

$$
\mathcal{L}_{x, y}=\mathcal{L}_{\tau}(\varphi)=(\beta, y-x)
$$

where $(3 . \tau)$ is a mique solution of the system

$$
\left\{\begin{array}{c}
H^{\mu}(\beta)=0 \\
\tau \nabla H^{\mu}(\beta)=y-x \\
\tau>0 .
\end{array}\right.
$$

Proof. From the lemma 3.5.1 and lemma 3.5.2 it easily follows that if the optimal path from the point $x$ to the point $y$ exists then it is lincar. For any linear patls

$$
\varphi(t)=x+\frac{t}{\tau}(y-x)
$$

one has

$$
\mathcal{L}_{\tau}(\varphi)=\tau L^{\mu}\left(\frac{y-x}{\tau}\right)
$$


Therefore for an optimal path $\varphi:[0, \tau] \rightarrow R^{\mu}$

$$
\tau L^{\mu}\left(\frac{y-x}{\tau}\right)=\inf _{t>0}\left\{t L^{\mu}\left(\frac{y-x}{t}\right)\right\}
$$

Let us note that the function $t L^{\mu}\left(\frac{y-x}{t}\right)$ it convex with respect to $t$ for any $y \neq x$ and

$$
t L^{\prime \prime}\left(\frac{y-x}{t}\right) \rightarrow \infty \text { as } t \rightarrow 0 \text { or } t \rightarrow \infty
$$

From this it follows that $\tau$ is a unique solution of the equation

$$
\frac{d}{d t}\left(t L^{\mu}\left(\frac{y-x}{t}\right)\right)=0
$$

It is casy to see that $t=\tau$ is a solution of the equation $(50)$ if $(\tau, \beta)$, where $3=\Gamma^{\prime \prime}\left(\frac{y-r}{r}\right)$. is a solution of the system (49). Due to the convexity of the function $H^{\prime \prime}(\cdot)$ this system has a unique solution. From this it follows the theorem 3.5.1.

Let us consicler now the case $\nu=1$.Consider the following equation

$$
\partial_{0} H_{\{1\}}^{1, \mu}(\alpha, \beta)=0
$$

For any 3 there exists a unique solution $\left(\alpha_{0}(\beta), \beta\right)$ of the equation $(51)$. Note that for any $\beta$ for which

$$
H_{\{1\}}^{1, \mu}\left(\alpha_{0}(\beta), \beta\right)<H_{\{\emptyset\}}^{1, \mu}\left(\alpha_{0}(\beta), \beta\right)
$$

there exists a unicue solution $\left(\alpha_{1}(\beta), \beta\right)$ of the system

$$
\left\{\begin{array}{l}
H_{\{1\}}^{1, \mu}(\alpha, \beta)=H_{\{\emptyset\}}^{1, \mu}(\alpha, \beta) \\
\partial_{i 1} H_{\{1\}}^{1, \mu}(\alpha, \beta)<0
\end{array}\right.
$$

Consider the linetion

$$
\mathcal{H}_{\forall}^{1, \mu \prime}(\beta)= \begin{cases}H_{\{1\}}^{1, \mu \prime}\left(\alpha_{0}(\beta), \beta\right) & \text { if } H_{\{1\}}^{1, \mu}\left(\alpha_{0}(\beta), \beta\right) \geq H_{\{\emptyset\}}^{1, \mu \prime}\left(\alpha_{0}(\beta) . \beta\right) \\ H_{\{1\}}^{1, \mu,}\left(\alpha_{1}(\beta), \beta\right) & \text { otherwise. }\end{cases}
$$


Theorem 3.5.2 Let $\nabla \mathcal{H}_{\emptyset}^{1.4 t}(0) \neq 0$. Then for any $x=\left(x^{0}, \bar{x}\right) . y=\left(y^{0} \cdot \bar{y}\right) \in$ $R_{+}^{1} \times R^{\mu}$ such that $x^{0}=y^{0}=0$ and $\bar{x} \neq \bar{y}$ there crists the unique optimal path $\rho:[0, \tau] \rightarrow R_{+}^{1} \times R^{\mu}$ from $x$ to $y$, this optimal path is limar

$$
\vartheta(t)=x+\frac{t}{\tau}(y-x)
$$

and

$$
\mathcal{L}_{x \cdot y}=\mathcal{L}_{\tau}(\varphi)=(3 . \bar{y}-\bar{x})
$$

where (3.T) is a unique solution of the system

$$
\left\{\begin{array}{l}
\mathcal{H}_{\emptyset}^{1 \cdot \mu}(\xi)=0 . \\
\tau \Gamma^{\prime} \mathcal{H}_{\emptyset}^{1 \cdot \mu}(\beta)=\bar{y}-\bar{x}
\end{array}\right.
$$

To prow this theorem note first that for any linear path $\varphi:[0 . \tau] \rightarrow$ $R_{+}^{\prime \prime} \times R^{\prime \prime}$ such that $\hat{r}^{\prime}(t)=\left(\hat{r}^{0}(t) \cdot \bar{y}(t)\right), \hat{\varphi}^{0}(t)=0$. we have

$$
\dot{L}(\hat{\tau})=\tau L_{\otimes}\left(\frac{\varphi(\tau)-\varphi(0)}{\tau} .\right.
$$

where $L_{\emptyset}(\cdot)$ is the Legendre transform of the function $\mathcal{H}_{b}^{1, \mu}(\cdot)$. Then it is sufficient 10 weat the proof of the theorem 3.5.1.

For arbitrary $x, y \in R_{0}^{1} \times R^{u}$ one can easily get the following theorem.

Theorem 3.5.3 let $\Gamma \mathcal{H}_{\emptyset}^{1, \mu}(0) \neq 0$. Then for any $x=\left(x^{0}, \bar{x}\right), y=\left(y^{0}, \bar{y}\right)$ there exists an optimal path $\mathrm{\varphi}:[0 . \tau] \rightarrow R_{+}^{1} \times R^{\mu}$ from $x$ to $y$, this optimal path is piere winelimere.

$$
r(t)= \begin{cases}x+\frac{t}{\tau_{1}}\left(x_{1}-x\right) & \text { for } 0 \leq t \leq \tau_{1} \\ x_{1}+\frac{t-\tau_{1}}{\tau_{2}}\left(x_{2}-x_{1}\right) & \text { for } \tau_{1} \leq t \leq \tau_{1}+\tau_{2} \\ r_{2}+\frac{t-\tau_{2}-\tau_{1}}{\tau_{3}}\left(y-x_{2}\right) & \text { for } \tau_{1}+\tau_{2} \leq t \leq \tau_{1}+\tau_{2}+\tau_{3}\end{cases}
$$

where $x_{1}=\left(0 . \bar{r}_{1}\right) \cdot x_{2}=\left(0 . \bar{x}_{2}\right)$. nud

$$
\dot{L}_{x, y}=\tau_{1} L_{\{1\}}^{1, \mu}\left(\frac{x_{1}-x^{\prime}}{\tau_{1}}\right)+\tau_{2} L_{\emptyset}^{1 . \mu}\left(\frac{\bar{x}_{2}-\bar{x}_{1}}{\tau_{2}}\right)+\tau_{3\}} L_{\{1\}}^{1, \mu}\left(\frac{y-x_{2}}{\tau_{3}}\right)=
$$




$$
=\inf _{t_{1} \geq 0, t_{2} \geq 0 . \tau_{3} \geq 0, \bar{s}_{1}, \bar{x}_{2}} t_{1} L_{\{1\}}^{1, \mu}\left(\frac{x_{1}-x}{t_{1}}\right)+t_{2} L_{\emptyset}\left(\frac{\bar{x}_{2}-\bar{x}_{1}}{t_{2}}\right)+t_{33} L_{\{1\}}\left(\frac{y-x_{2}}{t_{3}}\right)
$$

where $L_{\{1\}}(\cdot)$ is the Legendre transform of the function $H_{\{1\}}^{1, \mu}(\cdot) . L_{\emptyset}(\cdot)$ is the Legendie trunsform of the function $\mathcal{H}_{\emptyset}^{1 . \mu}(\cdot)$.

Let us consider the case $\nu=\mu=1$. We shall assume that

$$
\frac{d}{d \beta} \mathcal{H}_{\emptyset}^{1.1}(0) \neq 0
$$

Then from the construction of the function $\mathcal{H}_{\emptyset}^{1.1}(0)$ it follows that

$$
\nabla H_{\{1\}}^{1.1}(0.0) \neq 0 \text {. }
$$

Consider the following equation

$$
\mathcal{H}_{\emptyset}^{1.1}(;)=0
$$

Due to (5-1) this equation has exactly two different real solutions, $\beta_{1} \leq 0 \leq$ $\beta_{2}, \quad \beta_{1} \neq \beta_{2}$. C'onsider also the system

$$
\left\{\begin{array}{l}
H_{\{1\}}^{1,1}(\alpha, \beta)=0 \\
\tau \Gamma H_{\{1\}}^{1.1}(a, \beta)=x \\
\tau>0 .
\end{array}\right.
$$

For any $x=\left(x^{0}, x^{1}\right) \neq 0$ this sy stem has the unique solution $a^{*}\left(x^{*}\right) \cdot 3^{*}(x), \tau^{*}(x)$.

Theorem 3.5.4 let (.5.) be satisfied. Then for any $x=\left(x^{0} . x^{1}\right) \in R_{+}^{1} \times R^{1}$ the following condusions hold:

(i) l.t. $\beta_{1} \leq f^{*}(x) \leq 3_{2}$ then

$$
\dot{L}_{(0, x}=n^{*}(x) x^{0}+3^{*}(x) x^{1}
$$

and the optimal path from o to $x$ is linear

$$
r(t)=\frac{t}{\tau^{*}(x)} x, t \in\left[0, \tau^{*}(x)\right] .
$$


(ii) $1.11 \cdot 3 *(x)<3.16 \mathrm{cn}$

$$
\mathcal{L}_{0, x}=\alpha_{1} x^{0}+\beta_{1} x^{1}
$$

where $a_{1}$ is defined by the system

$$
\left\{\begin{array}{l}
H_{\{1\}}^{1,1}\left(\alpha_{1} \cdot \beta_{1}\right)=0 \\
\partial_{i x} H_{\{1\}}^{1,1}\left(\alpha_{1}, \beta_{1}\right)>0
\end{array}\right.
$$

and the optimal path from 0 to $x$ is piecewise linear

$$
F(l)= \begin{cases}\frac{t}{\tau_{-}^{\natural}}{ }_{-} & \text {if } 0 \leq t \leq \tau_{-}^{\emptyset} . \\ z_{-}+\frac{t-\tau_{-}^{\emptyset}}{\tau_{-}^{*}}\left(x-z_{-}\right) & \text {if } \tau_{-}^{\emptyset} \leq t \leq \tau_{-}^{\emptyset}+\tau_{-}^{*}\end{cases}
$$

where $z_{-}=\left(0, z_{-}^{1}\right) . z_{-}^{1}<0, \tau_{-}^{\emptyset}>0, \tau_{-}^{*}>0$ are defined by the systrm

$$
\left\{\begin{array}{l}
\nabla H_{\{1\}}^{1,1}\left(\alpha_{1}, \beta_{1}\right)=\frac{x-z_{-}}{\tau_{-}^{*}} \\
\partial_{\beta} \mathcal{H}_{\emptyset}^{1,1}\left(\beta_{1}\right)=\frac{z_{-}^{1}}{\tau_{-}^{\emptyset}}
\end{array}\right.
$$

(iii) let $: 3_{2}<33^{*}\left(x^{2}\right)$, then

$$
\dot{L}_{0, x}=\alpha_{2} x^{0}+\beta_{2} x^{1}
$$

where $\mathrm{a}_{2}$ is defincd by the system

$$
\left\{\begin{array}{l}
H_{\{1\}}^{1,1}\left(\alpha_{2}, \beta_{2}\right)=0 \\
\partial_{\omega} H_{\{1\}}^{1,1}\left(\alpha_{2} \cdot j_{2}\right)>0
\end{array}\right.
$$

and the optimal path from 0 to $x$ is pieceutise linear

$$
\hat{r}(1)= \begin{cases}\frac{t}{\tau_{+}^{\natural}} z_{+} & \text {if } 0 \leq t \leq \tau_{+}^{\emptyset} . \\ z_{+}+\frac{t-\tau_{+}^{\emptyset}}{\tau_{+}^{*}}\left(x-z_{+}\right) & \text {if } \tau_{+}^{\emptyset} \leq t \leq \tau_{+}^{\emptyset}+\tau_{+}^{*}\end{cases}
$$


where $z_{+}=\left(0, z_{+}^{1}\right) \cdot z_{+}^{1}>0, \tau_{+}^{\emptyset}>0, \tau_{+}^{*}>0$ are defined by the system

$$
\left\{\begin{array}{l}
\nabla M_{\{1\}}^{1,1}\left(\alpha_{2}, \beta_{2}\right)=\frac{x-z_{+}}{\tau_{+}^{*}}, \\
\frac{d}{d, 3} \mathcal{H}_{\emptyset}^{1.1}\left(\beta_{2}\right)=\frac{z_{+}^{1}}{\tau_{+}^{\emptyset}} .
\end{array}\right.
$$

Before starting the proof of the theorem 3.5 .4 let us give a geomotric interpretation to the conditions (i), (ii) and (iii) of this theorem. For this let. us introduce the polar coordinates in $R_{+}^{1} \times R^{1} \backslash\{0\}$.

$$
\begin{gathered}
x^{1}=r(x) \cos ;(x), \quad x^{0}=r(x) \sin \gamma(x) \\
r(x)>0, \quad 0 \leq \gamma(x) \leq \pi .
\end{gathered}
$$

Let us consider $1 w 0$ angles $12 .<\gamma_{1}$ such that

$$
\operatorname{ctg} \gamma_{1}=\frac{\partial_{\beta} H\left(\alpha_{1}, \beta_{1}\right)}{\partial_{\alpha} H\left(\alpha_{1}, \beta_{1}\right)}
$$

and

$$
\operatorname{ctg}_{12}=\frac{\partial_{\beta} H\left(\alpha_{2}, \beta_{2}\right)}{\partial_{\alpha} H\left(\alpha_{2}, \beta_{2}\right)}
$$

$i_{1}$ is the anglo between the positive direction of the axis $x^{1} \cdot\left(x^{0}=0\right)$ and the normal rector to the curve

$$
H(\alpha, \beta)=0
$$

at the point $\left(a_{1}, 3_{1}\right)$.

$\gamma_{2}$ is the anglo between the positive direction of the axis $x^{1}$ and the nomal vector to the culve

$$
H(\alpha, \beta)=0
$$

at the poim $\left(0_{2}, 3_{2}\right)$.

One can casily show that $0 \leq \gamma_{2}<\gamma_{1} \leq \pi$.

Let us note now that for any $x \in R_{+}^{1} \times R^{1}$

$$
\begin{aligned}
& 3^{*}(x)=\gamma_{1} \Longleftrightarrow \gamma(x)=\gamma_{1} . \\
& j^{*}(x)=\beta_{2} \Longleftrightarrow \gamma(x)=\gamma_{2} .
\end{aligned}
$$

and moresere

$$
\gamma_{1} \leq 3^{*}(x) \leq 3_{2} \Longleftrightarrow \gamma_{2} \leq \gamma(x) \leq \gamma_{1}
$$




$$
\begin{aligned}
& 3^{*}(x)<3_{1} \Longleftrightarrow \gamma(x)>\gamma_{1} . \\
& 3^{*}(x)>\beta_{2} \Longleftrightarrow \gamma(x)<\gamma_{2} .
\end{aligned}
$$

Due to the heorm 3.5.t for any $x \in R_{+}^{1} \times R^{1}$ the following conclusions hold.

(i) Let $\gamma_{2} \leq \gamma(x) \leq 7_{1}$. Then the optimal path from the point 0 to the point $x$ is lincin.

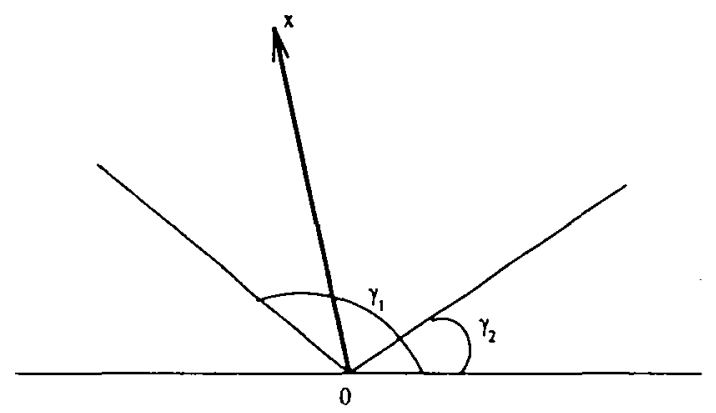

(ii) Ist $:(x)>i_{1}$. Then the optimal path from the point 0 to the point $x$ is the piecrwisclincar path $F$ consisting of two linear segments: the optimal path from $1010 z_{-}=\left(0 . z_{-}^{1}\right)$ and the optimal path from $z_{-}$to $x$. where $z_{-}=\left(0 . z_{-}^{1}\right) . z_{-}^{1}<0$ is miquely clefined by the equality

$$
i(x-z)=\gamma_{1}
$$

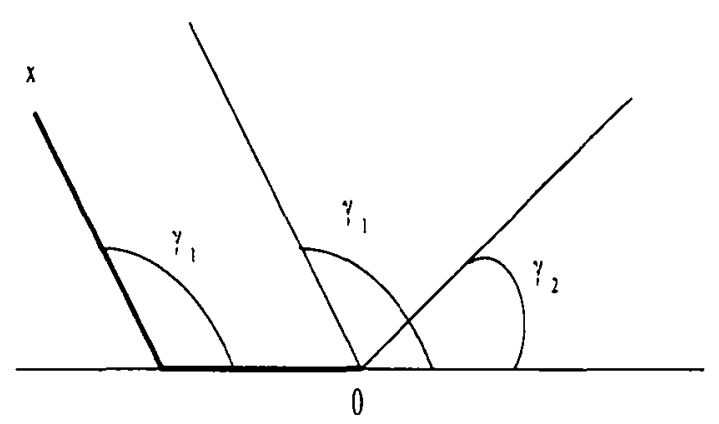

(iii) len $i(x)<i^{3}$. Then the optimal path from the point 0 to the point $x$ is ller picerewelinear path $p_{1}$ consisting of two linear segments: the optimal path from 0 to $z_{+}=\left(0 . z_{+}^{1}\right)$ and the optimal path from $z_{+}$to $x$. where $z_{+}=\left(0, z_{+}^{1}\right) . z_{+}^{1}<0$ is uniquely defined by the equality

$$
i(x-z)=92
$$




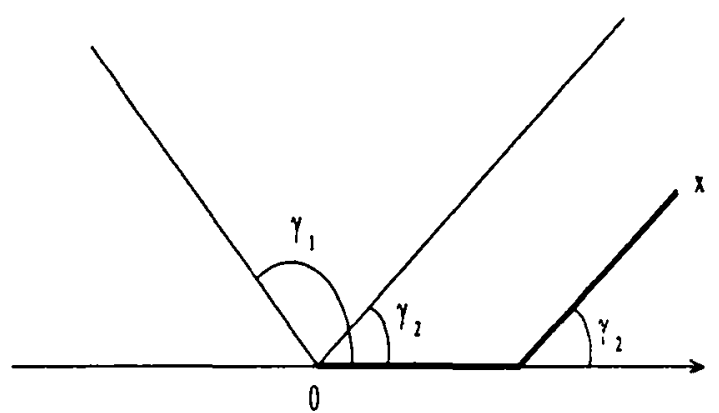

Proof of theorem 3.5.4. For any $x=\left(x^{0}, x^{1}\right)$ for which $x^{0}=0$ this theorem easily follows from the theorem 3.5.2.

Consider $x=\left(x^{0} . x^{1}\right) \in R_{+}^{1} \times R^{1}, x^{0} \neq 0$. Due to the theorem 3.5.3 there exists a pieceriselinear optimal path from 0 to $x$

$$
\gamma(t)= \begin{cases}\frac{t}{\tau^{\natural}} z & \text { if } 0 \leq t \leq \tau^{\emptyset} . \\ z+\frac{t-\tau^{\emptyset}}{\tau^{*}}(x-z) & \text { if } \tau^{\emptyset} \leq t \leq \tau^{\emptyset}+\tau^{*}\end{cases}
$$

and

$$
\begin{gathered}
\mathcal{L}_{0 . x}=\tau^{\emptyset} L_{\{\emptyset\}}^{1.1}\left(\frac{\tilde{z}^{1}}{\tau^{\emptyset}}\right)+\tau^{*} L_{\{1\}}^{1,1}\left(\frac{x-z}{\tau^{*}}\right)= \\
=\inf _{t_{1} \geq 0 . t_{2} \geq 0 . \approx=\left(0 . \sim^{1}\right)} t_{1} L_{\emptyset}^{1,1}\left(\frac{\tilde{z}^{1}}{t_{1}}\right)+t_{2} L_{\{1\}}^{1.1}\left(\frac{x-z}{t_{2}}\right)
\end{gathered}
$$

where $z_{-}=\left(0 . z^{1}\right) . \quad \tau^{\infty} \geq 0 . \tau^{*} \geq 0$ are defined by $(58)$.

Consider for any $z=\left(0, z^{1}\right) \neq 0$

$$
F(z)=\inf _{t_{1}>0 . t_{2}>0}\left\{t_{1} L_{\emptyset}\left(\frac{z^{1}}{t_{1}}\right)+t^{2} L_{\{1\}}^{1.1}\left(\frac{x-z}{t_{2}}\right)\right\}
$$

and

$$
F(0)=\inf _{t \geq 0}\left\{t L_{\{1\}}^{1.1}\left(\frac{x}{t}\right)\right\}
$$

Then

$$
\dot{\mathcal{L}}_{0 . .}=\inf _{z=\left(0 . z^{1}\right)} F(z)
$$

One can easily show that

$$
F(0)=\tau^{*}(x) L_{\{1\}}^{1.1}\left(\frac{x}{\tau^{*}(x)}\right)=
$$




$$
=\alpha^{*}(x) x^{0}+\beta^{*}(x) x^{1}
$$

and

$$
\begin{aligned}
H(z) & =\tau^{\emptyset}(z) L_{i \emptyset}\left(\frac{z^{1}}{\tau^{\emptyset}(z)}\right)+\tau^{*}(x-z) L_{\{1\}}^{1,1}\left(\frac{x-z}{\tau^{*}(x-z)}\right)= \\
& =\beta^{\natural}(z) z^{1}+\alpha^{*}(x-z) x^{0}+\beta^{*}(x-z)\left(x^{1}-z^{1}\right)
\end{aligned}
$$

where $a^{*}(x-z), z^{*}(x-z), \tau^{*}(x-z)$ is a unique solution of the system

$$
\left\{\begin{array}{l}
H_{\{1\}}^{1,1}(\alpha, \beta)=0 \\
\nabla H_{\{1\}}^{1,1}(\alpha . \beta)=\frac{x-z}{\tau}
\end{array}\right.
$$

and $3^{\emptyset}(z) . \tau^{n}(z)$ is a unique solution of the system

$$
\left\{\begin{array}{l}
\mathcal{H}_{\{\emptyset\}}^{1,1}(\beta)=0 \\
\frac{d}{d \beta} \mathcal{H}_{\{\emptyset\}}^{1.1}(\beta)=\frac{z^{1}}{\tau}
\end{array}\right.
$$

Note that

$$
\beta^{0}(z)= \begin{cases}\beta_{1} & \text { if } z^{1}>0 \\ \beta_{2} & \text { if } z^{1}<0\end{cases}
$$

Therefore

$$
F(z)= \begin{cases}3_{1} z^{1}+0^{*}(x-z) x^{0}+\beta^{*}(x-z)\left(x^{1}-z^{1}\right) & \text { if } z^{1}<0 \\ n^{*}(x) x^{0}+\xi^{*}(x) x^{1} & \text { if } z=0 \\ \beta_{2} z^{1}+\alpha^{*}(x-z) x^{0}+\xi^{*}(x-z)\left(x^{1}-z^{1}\right) & \text { if } z^{1}>0\end{cases}
$$

Note also that

$$
y^{*}(x-z) \rightarrow \overline{3}>0 \text { as } z=\left(0 . z^{1}\right), z^{1} \rightarrow-\infty
$$

and

$$
j^{*}(x-z) \rightarrow .3<0 \text { as } z=\left(0 . z^{1}\right) \cdot z^{1} \rightarrow+x
$$


where $(\underline{0} . \underline{3}),(\bar{n} \cdot \overline{3})$ are the two different solutions of the system

$$
\left\{\begin{array}{l}
H_{\{1\}}^{1,1}(\alpha, \beta)=0 \\
\partial_{\alpha} H_{\{1\}}^{1,1}(\alpha, \beta)=0 .
\end{array}\right.
$$

It is easy to see that the function $F(\cdot)$ is continuous and it is convex on each of the sets $\left\{z^{1} \leq 0\right\}$ and $\left\{z^{1} \geq 0\right\}$, but it should not be convex on $l^{1}$ .Therefore

$$
F(z) \rightarrow \infty \text { as } z^{1} \rightarrow \pm \infty
$$

So to get the infimum ( 5 i ) we must consider

$$
F_{-}^{\prime}(0)=\lim _{z \rightarrow 0-} \frac{d}{d z^{1}} F(z)=\beta_{1}-\beta^{*}(x)
$$

and

$$
F_{+}^{\prime}(0)=\lim _{z \rightarrow 0+} \frac{d}{d z^{1}} F(z)=\beta_{2}-\beta^{*}(x)
$$

Let us consider three cases.

(i) Let $\xi_{1} \leq j^{*}(x) \leq \beta_{2}$. Then $F_{-}^{\prime}(0) \leq 0$ and $F_{+}^{\prime}(0) \geq 0$.

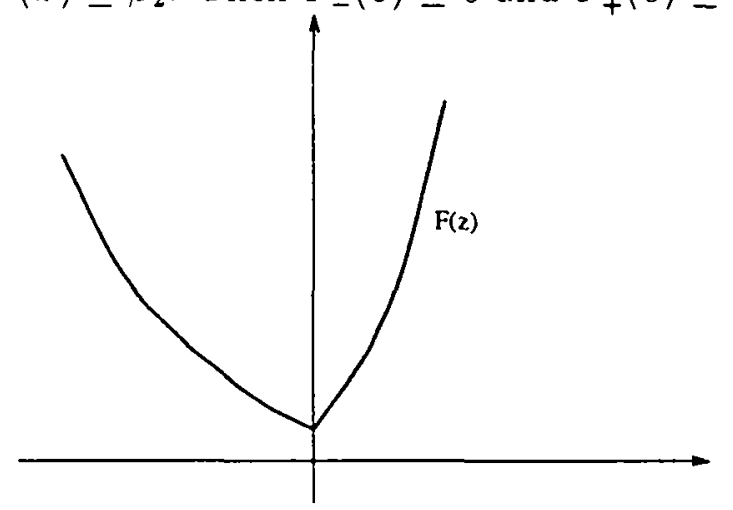

In this ase the function $F(\cdot)$ is convex on $R^{1}$ and it has a unique infimum in the point $z=0$.

(ii) Ject $f^{*}(x)<3_{1}$. Then $F_{-}^{\prime \prime}(0)>0$, and $F_{+}^{\prime}(0) \geq 0$. 


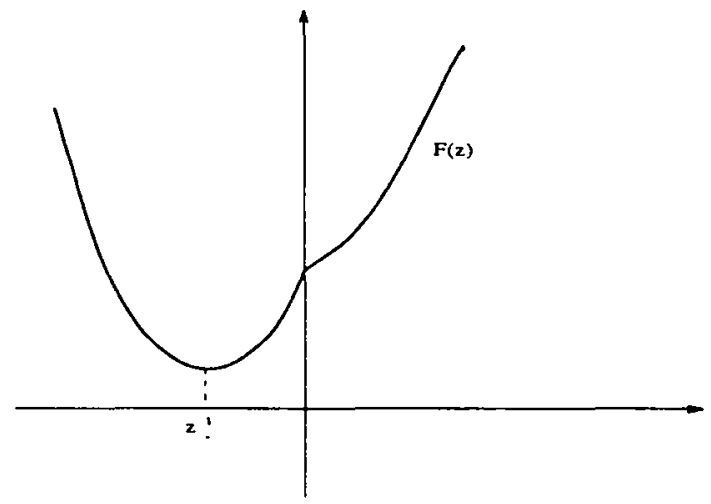

In this casc the function $F(\cdot)$ has a unique infimum in the point $z_{-}=$ $\left(0, z_{-}^{1}\right), z_{-}^{1}<0$ which is clefined by the following equation

$$
\xi^{*}(x-z)=3_{1} . \quad z=\left(0 . z_{-}^{1}\right) .
$$

(iii) Let $x_{2}<f^{*}(x)$. Then $F_{-}^{\prime}(0) \leq 0$. and $F_{+}^{\prime}(0)<0$.

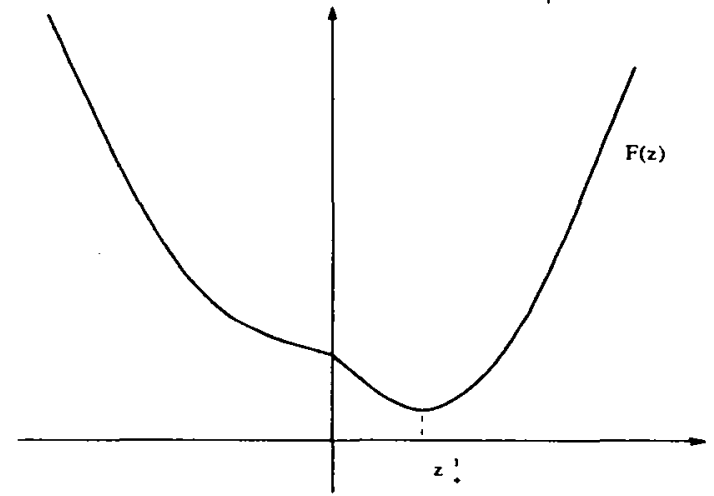

In this case the function $F(\cdot)$ has a unique infimum in the point $z_{+}=$ $\left(0 . z_{+}^{1}\right) . z_{+}^{1}>0$ which is defined by the following equation

$$
f^{*}(x-z)=\beta_{2} . z=\left(0, \tilde{z}_{+}^{1}\right) .
$$

In the first case

$$
\dot{\mathcal{L}}_{\text {U.x }}=F(0)=\alpha^{*}(x) x^{0}+\zeta^{*}(x) x^{1}
$$

and the oprimal path from () to $x$ is linear

$$
\vartheta(1)=\frac{t}{\tau^{*}(x)} x . t \in\left[0, \tau^{*}\right]
$$


In the secourd casce

$$
\mathcal{L}_{\text {U.J }}=\alpha_{1} x^{0}+\beta_{1} x^{1}
$$

where $0_{1}$ is defined by the system ( 56 ) and the optimal path from 0 to $r$ is piocenise linear

$$
\tau(l)= \begin{cases}\frac{t}{\tau_{-}^{\emptyset}} z_{-} & \text {if } 0 \leq t \leq \tau_{-}^{\emptyset} . \\ z_{-}+\frac{t-\tau_{-}^{\emptyset}}{\tau_{-}^{*}}\left(x-z_{-}\right) & \text {if } \tau_{-}^{\emptyset} \leq t \leq \tau_{-}^{\emptyset}+\tau_{-}^{*}\end{cases}
$$

where $z_{-}=\left(0 . z_{-}^{1}\right) . z_{-}^{1}<0 . \tau_{-}^{\emptyset}>0 . \tau_{-}^{*}>0$ are defined by the system

$$
\left\{\begin{array}{l}
\Gamma H_{\{1\}}^{1.1}\left(0_{1}, \beta_{1}\right)=\frac{x-z_{-}}{\tau_{-}^{*}} . \\
\partial_{3 .} \mathcal{H}_{\emptyset}^{1.1}\left(\beta_{1}\right)=\frac{z-\frac{1}{\emptyset}}{\tau_{-}^{\natural}}
\end{array}\right.
$$

In the case (iii)

$$
\dot{L}_{(j, x}=\alpha_{2} x^{0}+\beta_{2} x^{1}
$$

where $1_{2}$ is defined by the system ( $\left.5 i\right)$ and the optimal path from 0 to $x$ is piecervise lincar

$$
r(1)= \begin{cases}\frac{t}{\tau_{+}^{\emptyset}}{ }_{+} & \text {if } 0 \leq t \leq \tau_{+}^{\emptyset} . \\ i_{+}+\frac{t-\tau_{+}^{\emptyset}}{\tau_{+}^{\alpha}}\left(x-z_{+}\right) & \text {if } \tau_{+}^{\emptyset} \leq t \leq \tau_{+}^{\emptyset}+\tau_{+}^{*}\end{cases}
$$

where $\tau_{+}=\left(0 . z_{+}^{1}\right) \cdot z_{+}^{1}>0 . \tau_{+}^{0}>0 . \tau_{+}^{*}>0$ are defined by the system

$$
\left\{\begin{array}{l}
\nabla I_{\{1\}}^{1.1}\left(n_{2} .3_{2}\right)=\frac{x-z_{+}}{\tau_{+}^{*}} \\
\partial_{3} \mathcal{H}_{\emptyset}^{1.1}\left(3_{2}\right)=\frac{z_{+}^{1}}{\tau_{+}^{\phi}}
\end{array}\right.
$$

The theorem 3.j. is is proved. 
Theorem 3.5.5 Let (.54) be sutisfied. Then for any $x=\left(x^{0}, x^{1}\right) \in R_{+}^{1} \times R^{1}$ the follouing conchusions hold.

(i) let $: 3_{1} \leq 3 *(-x) \leq 3_{2}$ then

$$
\dot{L}_{2,0}=-\alpha^{*}(-x) \cdot x^{0}-\beta^{*}\left(-x^{\prime}\right) \cdot x^{\prime}
$$

and the optimal path from $x$ to 0 is linear.

$$
\varphi(t)=x-\frac{t}{\tau^{*}(-x)} x, t \in\left[0, \tau^{*}(-x)\right]
$$

(ii) $\operatorname{Lrt} ; *(-x)<\beta_{1}$, then

$$
\mathcal{L}_{x \cdot 0}=-\alpha_{1}^{\zeta} x^{0}-\beta_{1} x^{1}
$$

where $n_{1}^{6}$ is defined by the system

$$
\left\{\begin{array}{l}
H_{\{1\}}^{1,1}\left(\alpha_{1}^{\zeta}, \beta_{1}\right)=0, \\
\partial_{, 2} H_{\{1\}}^{1,1}\left(\alpha_{1}^{\zeta}, \beta_{1}\right)<0
\end{array}\right.
$$

and the optimal path from $x$ to 0 is pieceuise linear

$$
\gamma(t)= \begin{cases}x+\frac{t}{\tau_{+}^{*}}\left(z_{+}-x\right) & \text { if } 0 \leq t \leq \tau_{+}^{*} . \\ z_{+}-\frac{t-\tau_{+}^{*}}{\tau_{+}^{\emptyset}} z_{+} & \text {if } \tau_{+}^{*} \leq t \leq \tau_{+}^{\triangleright}+\tau_{+}^{*}\end{cases}
$$

where $z_{+}=\left(0, z_{+}^{1}\right), z_{+}^{1}>0, \tau_{+}^{\emptyset}>0, \tau_{+}^{*}>0$ are defined by the system

$$
\left\{\begin{array}{l}
\Gamma H_{\{1\}}^{1,1}\left(\alpha_{1}^{\zeta}, \beta_{1}\right)=\frac{z_{+}-x}{\tau_{+}^{*}} \\
\partial_{3} \mathcal{H}_{\emptyset}^{1.1}\left(\beta_{1}\right)=\frac{-z_{+}^{1}}{\tau_{+}^{\emptyset}}
\end{array}\right.
$$

(iii) $\left[+1 \cdot 3_{2}<3^{*}(-x)\right.$. $1 / 6 n$

$$
\mathcal{L}_{r .0}=-\alpha_{2}^{6} x^{0}-3_{2} \cdot r^{1}
$$


where $a_{2}^{5}$ is defined by the system

$$
\left\{\begin{array}{l}
I_{\{1\}}^{1,1}\left(\alpha_{2}^{\zeta}, \beta_{2}\right)=0 \\
\partial_{1} H_{\{1\}}^{1.1}\left(\alpha_{2}^{\zeta}, \beta_{2}\right)<0
\end{array}\right.
$$

and the oplimal path from $x$ to 0 is pieceutise linear.

$$
\hat{r}(l)= \begin{cases}x+\frac{t}{\tau_{-}^{*}}\left(z_{-}-x\right) & \text { if } 0 \leq t \leq \tau_{-}^{*}, \\ z_{-}-\frac{t-\tau_{-}^{*}}{T_{-}^{\emptyset}} z_{-} & \text {if } \tau_{-}^{*} \leq t \leq \tau_{-}^{\emptyset}+\tau_{-}^{*}\end{cases}
$$

where $z_{-}=\left(0 . z_{-}^{1}\right) . z_{-}^{1}<0 . \tau_{-}^{n}>0 . \tau_{-}^{*}>0$ are defined by the system

$$
\left\{\begin{array}{l}
\Gamma H_{\{1\}}^{1.1}\left(\alpha_{2}^{6}, \beta_{2}\right)=\frac{z_{-}-x}{\tau_{-}^{*}} \\
\dot{\partial}_{3} \mathcal{H}_{\emptyset}^{1.1}\left(\beta_{2}\right)=-\frac{-z_{-}^{1}}{\tau_{-}^{\emptyset}}
\end{array}\right.
$$

One can easily prove this theorem using the same argument as in the proof of the theorem 3.5.4.

\subsection{Stationary probabilities for an ergodic random walk in $Z_{+}^{2}$}

Let us consider a random walk $S_{t}(i, j), t \in Z_{+}$in $Z_{+}^{2}$. starting at the point (i.j) $\in Z_{+}^{\prime}:$

$$
\check{S}_{0}(i, j)=(i, j)
$$

and having lle transition probabilities as defined in the scction 2.2. Wr do not assume here that $d=1$.

C'onsider llac following functions

$$
\begin{aligned}
& M(a, 3)=\log \left\{\sum_{(i, j)} p_{i j} \exp \{a i+i j\}\right\} \\
& h_{1}(a .3)=\log \left\{\sum_{(i, j))} p_{i j}^{\prime} \exp \{a i+i j\}\right\}
\end{aligned}
$$




$$
h_{2}(a, 3)=\log \left\{\sum_{i, j} p_{i j}^{\prime \prime} \exp \{a i+, j j\}\right\}
$$

For any a let us consider $\beta_{0}(\alpha)$ such that

$$
\partial_{i 3} H\left(\alpha, \beta_{0}(\alpha)\right)=0 .
$$

and for any 3 let us consider $a_{0}(, 3)$ such that

$$
\partial_{0} H\left(\alpha_{0}(\beta), \beta\right)=0 \text {. }
$$

Consider

$$
\mathcal{H}_{\{1\}}(\alpha)= \begin{cases}H\left(\alpha . \xi_{0}(\alpha)\right) & \text { if } H\left(\alpha \cdot \beta_{0}(\alpha)\right) \geq h_{1}\left(\alpha, \xi_{0}(\alpha)\right) \\ H(\alpha, \beta(\alpha)) & \text { otherwise. }\end{cases}
$$

where $3(0)$ is dofined from the system

$$
\left\{\begin{array}{c}
H(a, 3)=h_{1}(\alpha, \beta(a)) \\
\partial_{i 3} H(\alpha .3(a))<0
\end{array}\right.
$$

Consider also

$$
\left.\mathcal{H}_{\{2\}}(.\}\right)= \begin{cases}H\left(\alpha_{0}(3) . \beta\right) & \text { if } H\left(\alpha_{0}(\beta), \beta\right) \geq h_{2}\left(\alpha_{0}(\beta), \beta\right) \\ H(\alpha(3) .3) & \text { otherwise, }\end{cases}
$$

where $a(3)$ is clofined from the system

$$
\left\{\begin{array}{c}
H(\alpha(3), \beta)=h_{2}(\alpha(\beta), \beta) \\
\partial_{1} H(\alpha(\beta), \beta)<0
\end{array}\right.
$$

Let $L(\cdot) . I_{1}(\cdot) . L_{2}(\cdot)$ be the Legendre transforms of the functions $H(\cdot), \mathcal{H}_{\{1\}}(\cdot), \mathcal{H}_{\{2\}}(\cdot)$ correspondingly: Consider the following function

$$
I .((i . j) . v)= \begin{cases}L_{1.2}(v) & \text { if } i>0, j>0 \\ L_{1}\left(v^{1}\right) & \text { if } i>0, j=0 \\ L_{2}\left(v^{2}\right) & \text { if } i=0, j>0 \\ 0 & \text { if } i=j=0\end{cases}
$$


where $v=\left(1 \cdot 1 \cdot i^{2}\right)$.

From the large (leviation principle for the random walk in $Z_{+}^{1} \times Z^{1}(3.3 .1)$ it easily follow's the large deviation principle for an ergodic random walk in $Z_{+}^{2}$. For the transient case in the quarter plane there is an open problem for the path irlcutically erpual to $(0.0)$. We do not touch this case in our paper.

Theorem 3.6.1 Let the random walk $S$, be ergodic. Then it satisfies the large deviation principle with the action functionals $\mathcal{L}_{r}$ such that for any $\tau \geq 0$ and for an!y continuous path

$$
\begin{gathered}
\vartheta:[0 . \tau] \rightarrow R_{+}^{2} \\
\mathcal{L}_{+}(\varphi)=\int_{0}^{\tau} L(\varphi(t), \dot{\varphi}(t)) d t
\end{gathered}
$$

if the path is absolutely continuous, and

$$
\mathcal{L}_{\tau}(\varphi)=\infty
$$

otherwist.

Let the andom walk $s$, be ergodic. C'onsider the stationary probabilities $\pi(x), x \in Z_{+}^{2}$ of this random walk. Ising the large cleviation principle we shall get now a logarithmic asymptotics of $\pi\left(\left[x N^{j}\right]\right)$ as $N \rightarrow \infty$ for any $x \in R_{+}^{2}$.

We shall assume here that.

$\left(H_{0}\right)$ :

(0) The Markos chain corresponding to the random walk s, is irreducible and aperiodic.

(1) The induced Markov chain with the set of states $Z_{+}$and transition probabilities

$$
p_{1}\left(l . l^{\prime}\right)=\sum_{h^{\prime}} p\left((1 . l) \rightarrow\left(1+h^{\prime} . l^{\prime}\right)\right)
$$

is irreducible and aperiodic:

(2) The same for the other incluced Markor chain with transition probat bilities

$$
p_{2}\left(k \cdot k^{\prime}\right)=\sum_{l^{\prime}} p\left((k, 1) \rightarrow\left(k^{\prime} .1+l^{\prime}\right)\right)
$$

$(H)$ :

$$
\Gamma H(0.0) \neq 0 \text {. }
$$




$$
\begin{aligned}
& \frac{d}{d \alpha} \mathcal{H}_{\{1\}}(0) \neq 0 \\
& \frac{d}{d \beta} \mathcal{H}_{\{2\}}(0) \neq 0
\end{aligned}
$$

Using the same arguments as in the section 3.5 one can easily show that for any two point there exists an optimal path from one point to another. Consider for any $x \in R_{+}^{2}$ an optimal path to go from the point 0 to the point $x$

$$
\varphi_{x}:\left[0, \tau_{x}\right] \rightarrow R_{+}^{2}
$$

and consider

$$
\mathcal{L}_{0, x}=\mathcal{L}_{\tau_{x}}\left(\varphi_{x}\right)
$$

Theorem 3.6.2 For any $x \in R_{+}^{2}$

$$
\lim _{N \rightarrow \infty} \frac{1}{N} \log \pi([x N])=-\mathcal{L}_{0, x}
$$

Proof.

It's easy to see that:

$$
\pi([x N]) \geq \pi(0) P\left(S_{N_{\tau}}(0)=[x N]\right)
$$

Using the lower bound for the last factor we obtain the following bound:

$$
\frac{1}{N} \log \pi([x N]) \geq-\mathcal{L}_{0, x}-\varepsilon
$$

To get the upper bound:

$$
\frac{1}{N} \log \pi([x N]) \leq-\mathcal{L}_{0, x}+\varepsilon
$$

we consider the interval $[0, N r+N \tau]$ for some large $r$ and $\tau \geq \tau_{x}$, where $\tau_{2}$ is the time of optimal way from point 0 to point $x$.

Let $\gamma_{0}$ be the last moment of time when random walk hits 0 , such that

$$
\gamma_{0} \in[1,1+N r+N \tau]
$$

Then we have:

$$
P\left(S_{1+N r+N \tau}(0)=[x N]\right)=\sum_{k=1}^{N r} P\left(\gamma_{0}=k,\right.
$$




$$
\left.S_{1+N r+N \tau}(0)=[x N]\right)+P\left(\gamma_{0} \geq 1+N r, S_{1+N r+N \tau}(0)=[x N]\right)
$$

Let us note that for each term of first sum we have:

$$
\frac{1}{N} \log \left(P\left(\gamma_{0}=k, S_{1+N r+N \tau}(0)=[x N]\right) \leq-\mathcal{L}_{0, x}+\varepsilon\right.
$$

for $N \geq N(\varepsilon)$, and $N(\varepsilon)$ doesn't depend from $k$.In order to estimate the second term in (63) we note that for our case of ergodic random walk in quarter of plane the following bound takes place:

$$
P\left(\gamma_{0} \geq N r\right) \leq \exp (-\alpha r N)
$$

$-\alpha$ is some fixed constant. The upper bound (65) was proved in ([5]). Note that $r$ may be taken sufficiently large. Then keeping in mind exponential convergence to the stationary measure we can obtain the upper bound for $\frac{1}{N} \log \pi([x N])$ by simple reasoning. Thus the statement of theorem 3.6 .2 is proved.

Let us calculate for any $x \in R_{+}^{2}$ the value $\mathcal{L}_{0, x}$. For this let us consider the system

$$
\left\{\begin{array}{l}
H(\alpha, \beta)=0, \\
\tau \nabla H(\alpha, \beta)=x, \\
\tau>0
\end{array}\right.
$$

For any $x \in R_{+}^{2}$ there exists a unique solution of this system $\alpha^{*}(x), \beta^{*}(x), \tau^{*}(x)$.

Consider also the following equations

$$
\mathcal{H}_{\{1\}}(\alpha)=0
$$

and

$$
\mathcal{H}_{\{2\}}(\beta)=0
$$

Due to the convexity of the functions $\mathcal{H}_{\{1\}}(\cdot)$ and $\mathcal{H}_{\{2\}}(\cdot)$ from the assumptions $(H)$ it follows that each of these equations has exactly two different solutions. Let $\alpha_{\{1\}}^{1}<\alpha_{\{1\}}^{2}$ and $\beta_{\{2\}}^{1}<\beta_{\{2\}}^{2}$ be the solutions of the equations (67), and (68) correspondingly. One can easily show that

$$
\alpha_{\{1\}}^{1} \leq 0 \leq \alpha_{\{1\}}^{2}
$$




$$
\begin{gathered}
\beta_{\{2\}}^{1} \leq 0 \leq \beta_{\{2\}}^{2} \\
\frac{d}{d \alpha} \mathcal{H}_{\{1\}}\left(\alpha_{\{1\}}^{1}\right)<0, \frac{d}{d \alpha} \mathcal{H}_{\{1\}}\left(\alpha_{\{1\}}^{2}\right)>0
\end{gathered}
$$

and

$$
\frac{d}{d \beta} \mathcal{H}_{\{2\}}\left(\beta_{\{2\}}^{1}\right)<0, \quad \frac{d}{d \beta} \mathcal{H}_{\{2\}}\left(\beta_{\{2\}}^{2}\right)>0
$$

Theorem 3.6.3 For any $x \in R_{+}^{2}$ the following conclusions hold

(i) Let $\alpha^{*}(x) \leq \alpha_{\{1\}}^{2}$ and $\beta^{*}(x) \leq \beta_{\{2\}}^{2}$ then

$$
\mathcal{L}_{0, x}=\alpha^{*}(x) x^{1}+\beta^{*}(x) x^{2}
$$

and the optimal path from 0 to $x$ is linear

$$
\varphi_{x}(t)=\frac{t}{\tau^{*}(x)} x, \quad t \in\left[0, \tau^{*}(x)\right],
$$

(ii) Let $\alpha^{*}(x)>\alpha_{\{1\}}^{2}$ and $\beta^{*}(x) \leq \beta_{\{2\}}^{2}$ then

$$
\mathcal{L}_{0, x}=\alpha_{\{1\}}^{2} x^{1}+\beta_{\{1\}}^{2} x^{2}
$$

where $\beta_{\{1\}}^{2}$ is defined by the system

$$
\left\{\begin{array}{l}
H\left(\alpha_{\{1\}}^{2}, \beta_{\{1\}}^{2}\right)=0, \\
\partial_{\beta} H\left(\alpha_{\{1\}}^{2}, \beta_{\{1\}}^{2}\right) \cdot \geq 0
\end{array}\right.
$$

For $x^{2}=0$ the optimal path from 0 to $x$ is linear

$$
\varphi_{x}^{1}(t)=\frac{t}{\tau^{1}} x, t \in\left[0, \tau^{1}\right],
$$

where $\tau^{1}>0$ is defined from the equation

$$
\frac{d}{d \alpha} \mathcal{H}_{\{1\}}\left(\alpha_{\{1\}}^{2}\right)=\frac{x^{1}}{\tau^{1}} .
$$

For $x^{2}>0$ the optimal path from 0 to $x$ is piecewise linear

$$
\varphi_{x}^{1}(t)= \begin{cases}\frac{t}{\tau_{1}^{1}} z_{1} & \text { if } 0 \leq t \leq \tau_{1}^{1}, \\ z_{1}+\frac{t-\tau_{1}^{1}}{\tau_{1}^{2}}\left(x-z_{1}\right) & \text { if } \tau_{1}^{1} \leq t \leq \tau_{1}^{1}+\tau_{1}^{2}\end{cases}
$$


where $z_{1}=\left(z_{1}^{1}, 0\right), z_{1}^{1}>0, \tau_{1}^{1}>0, \tau_{1}^{2}>0$ are defined from the system

$$
\left\{\begin{array}{l}
\nabla H\left(\alpha_{\{1\}}^{2}, \beta_{\{1\}}^{2}\right)=\frac{x-z_{1}}{\tau_{1}^{2}} \\
\frac{d}{d \alpha} \mathcal{H}_{\{1\}}\left(\alpha_{\{1\}}^{2}\right)=\frac{z_{1}^{1}}{\tau_{1}^{2}}
\end{array}\right.
$$

(iii) Let $\alpha^{*}(x) \leq \alpha_{\{1\}}^{2}$ and $\beta^{*}(x)>\beta_{\{2\}}^{2}$ then

$$
\mathcal{L}_{0, x}=\alpha_{\{2\}}^{2} x^{1}+\beta_{\{2\}}^{2} x^{2}
$$

where $\alpha_{\{2\}}^{2}$ is defined by the system

$$
\left\{\begin{array}{l}
H\left(\alpha_{\{2\}}^{2}, \beta_{\{2\}}^{2}\right)=0, \\
\frac{\partial}{\partial \alpha} H\left(\alpha_{\{2\}}^{2}, \beta_{\{2\}}^{2}\right) \geq 0
\end{array}\right.
$$

For $x^{1}=0$ the optimal path from 0 to $x$ is linear

$$
\varphi_{x}^{2}(t)=\frac{t}{\tau^{2}} x, t \in\left[0, \tau^{2}\right]
$$

where $\tau^{2}>0$ is defined from the equation

$$
\frac{d}{d \beta} \mathcal{H}_{\{2\}}\left(\beta_{\{2\}}^{2}\right)=\frac{x^{2}}{\tau^{2}}
$$

For $x^{1}>0$ the optimal path from 0 to $x$ is piecewise linear

$$
\varphi_{x}^{2}(t)= \begin{cases}\frac{t}{\tau_{1}^{2}} z_{2} & \text { if } 0 \leq t \leq \tau_{1}^{2}, \\ z_{2}+\frac{t-\tau_{1}^{2}}{\tau_{2}^{2}}\left(x-z_{2}\right) & \text { if } \tau_{1}^{2} \leq t \leq \tau_{1}^{2}+\tau_{2}^{2}\end{cases}
$$

where $z_{2}=\left(0, z_{2}^{2}\right), z_{2}^{2}>0, \tau_{2}^{1}>0, \tau_{2}^{2}>0$ are defined from the system

$$
\left\{\begin{array}{l}
\nabla H\left(\alpha_{\{2\}}^{2}, \beta_{\{2\}}^{2}\right)=\frac{x-z_{2}}{\tau_{2}^{2}} \\
\frac{d}{d \beta} \mathcal{H}_{\{2\}}\left(\beta_{\{2\}}^{2}\right)=\frac{z_{2}^{2}}{\tau_{2}^{2}}
\end{array}\right.
$$


(iv) Let $\alpha^{*}(x)>\alpha_{\{1\}}^{2}$ and $\beta^{*}(x)>\beta_{\{2\}}^{2}$ then

$$
\mathcal{L}_{0, x}=\min \left\{\alpha_{\{1\}}^{2} x^{1}+\beta_{\{1\}}^{2} x^{2}, \alpha_{\{2\}}^{2} x^{1}+\beta_{\{2\}}^{2} x^{2}\right\}
$$

and the optimal path from 0 to $x$ is the following

$$
\varphi_{x}(t) \equiv \begin{cases}\varphi_{x}^{1}(t) & \text { if } \alpha_{\{1\}}^{2} x^{1}+\beta_{\{1\}}^{2} x^{2}>\alpha_{\{2\}}^{2} x^{1}+\beta_{\{2\}}^{2} x^{2} \\ \varphi_{x}^{1}(t) & \text { if } \alpha_{\{1\}}^{2} x^{1}+\beta_{\{1\}}^{2} x^{2}>\alpha_{\{2\}}^{2} x^{1}+\beta_{\{2\}}^{2} x^{2}\end{cases}
$$

One can easily prove this theorem using the same arguments as in the proof of the theorem 3.5.4.

It is useful to consider the following conditions which are equivalent to the conditions (i),(ii),(iii) and (iv) of the theorem 3.6.3

1. (i) $h_{1}\left(\alpha^{*}(x), \beta^{*}(x)\right) \leq 0, \quad h_{2}\left(\alpha^{*}(x), \beta^{*}(x)\right) \leq 0$.

2. $\sim$ (ii) $h_{1}\left(\alpha^{*}(x), \beta^{*}(x)\right)>0, \quad h_{2}\left(\alpha^{*}(x), \beta^{*}(x)\right) \leq 0$.

3. $\sim$ (iii) $h_{1}\left(\alpha^{*}(x), \beta^{*}(x)\right) \leq 0, \quad h_{2}\left(\alpha^{*}(x), \beta^{*}(x)\right)>0$.

4. $\sim$ (iv)' $h_{1}\left(\alpha^{*}(x), \beta^{*}(x)\right)>0, \quad h_{2}\left(\alpha^{*}(x), \beta^{*}(x)\right)>0$

This equivalence easily follows from the definition of the functions $\mathcal{H}_{\{1\}}$ and $\mathcal{H}_{\{2\}}$.

Let us give geometric interpretation to the conditions (i) - (iv). For this let us introduce the polar coordinates in $R_{+}^{2} \backslash\{0\}$.

$$
\begin{gathered}
x^{1}=r(x) \cos \gamma(x), \quad x^{2}=r(x) \sin \gamma(x) \\
r(x)>0, \quad 0 \leq \gamma(x) \leq \frac{\pi}{2} .
\end{gathered}
$$

Let us consider $0 \leq \gamma_{1} \leq \frac{\pi}{2}$ and $0 \leq \gamma_{2} \leq \frac{\pi}{2}$ such that

$$
\operatorname{tg} \gamma_{1}=\frac{\frac{\partial}{\partial \beta} H\left(\alpha_{\{1\}}^{2}, \beta_{\{1\}}^{2}\right)}{\frac{\partial}{\partial \alpha} H\left(\alpha_{\{1\}}^{2}, \beta_{\{1\}}^{2}\right)}
$$


and

$$
\operatorname{tg} \gamma_{2}=\frac{\frac{\partial}{\partial \beta} H\left(\alpha_{\{2\}}^{2}, \beta_{\{2\}}^{2}\right)}{\frac{\partial}{\partial \alpha} H\left(\alpha_{\{2\}}^{2}, \beta_{\{2\}}^{2}\right)}
$$

Note that for any $x \in R_{+}^{2}$

$$
\begin{aligned}
& \alpha^{*}(x)=\alpha_{\{1\}}^{2} \Longleftrightarrow \gamma(x)=\gamma_{1}, \\
& \beta^{*}(x)=\beta_{\{2\}}^{2} \Longleftrightarrow \gamma(x)=\gamma_{2},
\end{aligned}
$$

and moreover

$$
\begin{aligned}
& \alpha^{*}(x)<\alpha_{\{1\}}^{2} \Longleftrightarrow \gamma(x)>\gamma_{1}, \\
& \alpha^{*}(x)>\alpha_{\{1\}}^{2} \Longleftrightarrow \gamma(x)<\gamma_{1}, \\
& \beta^{*}(x)<\beta_{\{2\}}^{2} \Longleftrightarrow \gamma(x)<\gamma_{2}, \\
& \beta^{*}(x)>\beta_{\{2\}}^{2} \Longleftrightarrow \gamma(x)>\gamma_{2} .
\end{aligned}
$$

Due to the theorem 3.6 .3 for any $x \in R_{+}^{2}$ the following conclusions hold.

(i) Let $\gamma_{1} \leq \gamma(x) \leq \gamma_{2}$. Then the optimal path from the point 0 to the point $x$ is linear.

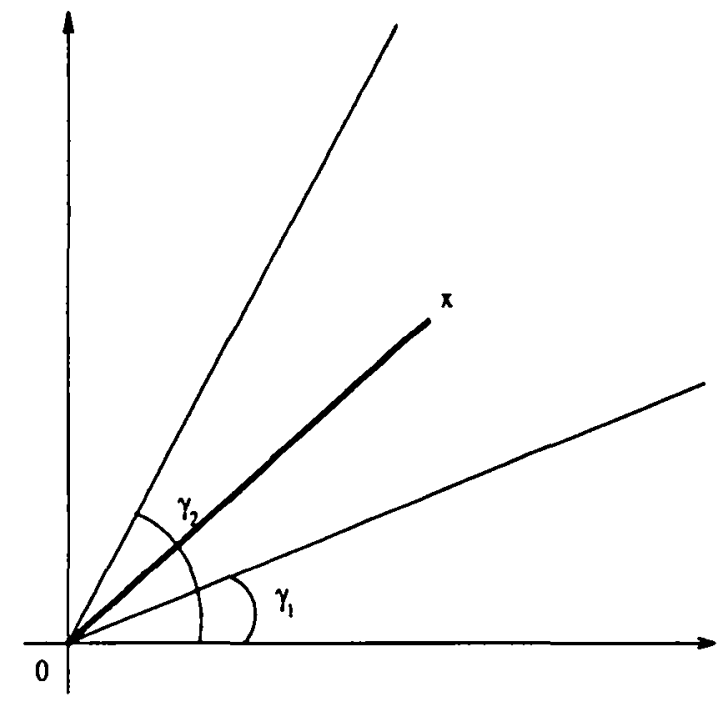

(ii) Let $\gamma(x)<\gamma_{1}$ and $\gamma(x) \leq \gamma_{2}$. Then the optimal path from the point 0 to the point $x$ is the piecewiselinear path $\varphi_{1}$ consisting of two linear segments: 
the optimal path from 0 to $z_{1}=\left(z^{1}, 0\right)$ and the optimal path from $z_{1}$ to $x$, where $z_{1}=\left(z^{1}, 0\right), z^{1}>0$ is uniquely defined by the equality

$$
\gamma\left(x-z_{1}\right)=\gamma_{1}
$$

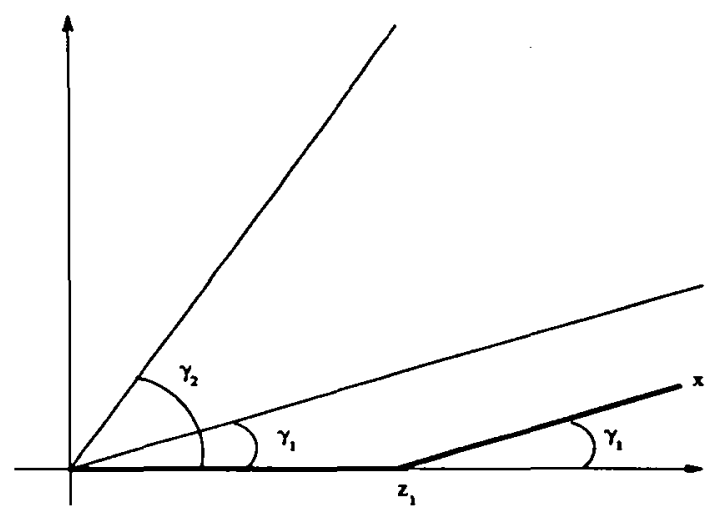

(iii) Let $\gamma(x) \geq \gamma_{1}$ and $\gamma(x)>\gamma_{2}$. Then the optimal path from the point 0 to the point $x$ is the piecewiselinear path $\varphi_{2}$ consisting of two linear segments: the optimal path from 0 to $z_{2}=\left(0, z^{2}\right)$ and the optimal path from $z_{2}$ to $x$, where $z_{2}=\left(0, z^{2}\right), z^{2}>0$ is uniquely defined by the equality

$$
\gamma\left(x-z_{2}\right)=\gamma_{2}
$$

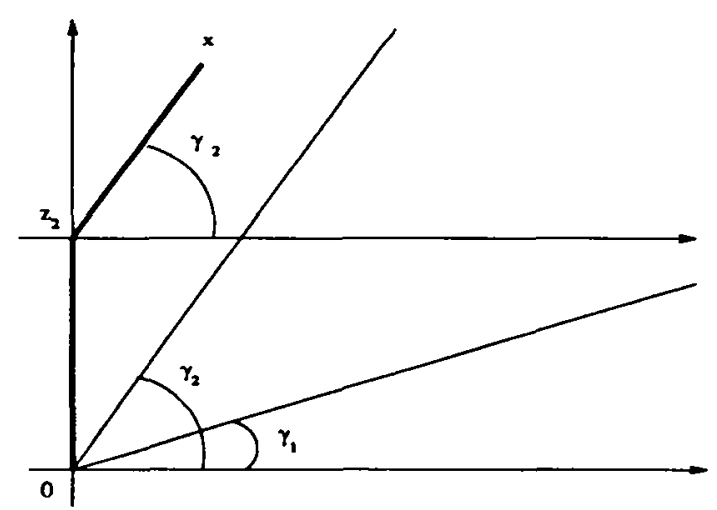

(iv) Let $\gamma(x)<\gamma_{1}$ and $\gamma(x)>\gamma_{2}$. Then the optimal path from the point 0 to the point $x$ is the path $\varphi_{x}^{1}$ or $\varphi_{x}^{2}$ which provides the minimum of the action functional. 


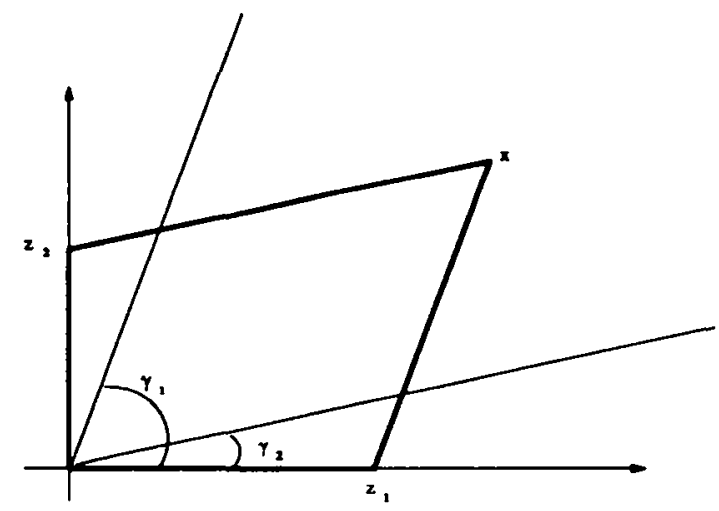

Define the sets

$$
\begin{gathered}
\mathcal{P}_{--}=\left\{x \in R_{+}^{2}: \gamma_{1} \leq \gamma(x) \leq \gamma_{2}\right\} \\
\mathcal{P}_{+-}=\left\{x \in R_{+}^{2}: \gamma(x)<\gamma_{1}, \gamma(x) \leq \gamma_{2}\right\} \\
\mathcal{P}_{-+}=\left\{x \in R_{+}^{2}: \gamma_{1} \leq \gamma(x), \gamma_{2}<\gamma(x)\right\} \\
\mathcal{P}_{++}=\left\{x \in R_{+}^{2}: \gamma(x)<\gamma_{1}, \gamma_{2}<\gamma(x)\right\}
\end{gathered}
$$

From theorem 3.6.3 it follows

Theorem 3.6.4 For any $x \in R_{+}^{2}$

$$
\frac{1}{N} \log \pi([N x]) \sim-\min \left\{\alpha_{\{1\}}^{2}(x) x^{1}+\beta_{\{1\}}^{2}(x) x^{2}, \alpha_{\{2\}}^{2}(x) x^{1}+\beta_{\{2\}}^{2}(x) x^{2}\right\}
$$

if $x \in \mathcal{P}_{++}$and

$$
\frac{1}{N} \log \pi([N x]) \sim \begin{cases}-\alpha^{*}(x) x^{1}-\beta^{*}(x) x^{2} & \text { if } x \in \mathcal{P}_{--} \\ -\alpha_{\{1\}}^{2}(x) x^{1}-\beta_{\{1\}}^{2}(x) x^{2} & \text { if } x \in \mathcal{P}_{+-} \\ -\alpha_{\{2\}}^{2}(x) x^{1}-\beta_{\{2\}}^{2}(x) x^{2} & \text { if } x \in \mathcal{P}_{-+}\end{cases}
$$

One can see that these results coincide with the results of the theorem 2.2.1. It is suggestive to find deeper connections between analytic and probabilistic approaches. 


\section{Appendix.}

\section{1}

Let us consider a countable Markov chain with a set of states $X$ and transition probabilities $p_{i, j}, i, j \in X$.

We shall assume that the following conditions are satisfied

$D$ :

(i) This Markov chain is irreducible and aperiodic.

(ii) There exist functions $f: X \rightarrow R_{+}$and $k: X \rightarrow Z_{+}$such that

(a) for any $b>0$

$$
\sum_{j \in X} \exp \{-b f(j)\}<\infty
$$

(b) there exists $d>0$ such that for any $i, j \in X$

$$
p_{i, j}=0 \text { if }|f(j)-f(i)|>d
$$

(c)

$$
\sup _{i} k(i)<\infty
$$

(d) there exists a finite subset $X_{0} \subset X$ such that for any $i \in X \backslash X_{0}$

$$
\sum_{j} p_{i, j}^{(k(i))} f(j) \leq f(i)-\epsilon
$$

where $\epsilon$ does not depend on $i \in X \backslash X_{0}$.

From these assumptions it follows (see [5] ) that this Markov chain is ergodic and moreover the two following propositions hold.

1. for any $h>0$ there exist $c>0$ and $h^{\prime}>0$ such that for any i.j $\in X^{\prime}$ and for any $t \in Z_{+}$

$$
p_{i, j}^{(t)} \leq c e^{h f(i)-h^{\prime} f(j)}
$$

2. for any $h>0$ there exist $c>0$ and $\alpha>0$, such that for any $i \in X^{\prime}$ and for any $t \in Z_{+}$

$$
\sum_{j \in X}\left|p_{i, j}^{(t)}-\pi(j)\right| \leq c e^{h f(i)-\alpha t}
$$

where $\pi(j), j \in X$ are the stationary probabilities for this Markov chain. 
Let consider a Markov chain having the set of states $Z_{+}$and transition probabilities $p_{i, j}, i, j \in Z_{+}$such that

$$
p_{i, j}=p_{j-i} \text { and } \sum_{j} p_{j-i}(j-i)<0
$$

for all $i>d_{1}$, and

$$
p_{i, j}=0 \text { if }|j-i|>d_{2}
$$

with some positive constants $d_{1}, d_{2}$. Then this Markov chain in ergodic and satisfies the conditions $D$ with the following functions

$$
f(j)=j \text { and } k(j)=1, j \in Z_{+}
$$

The Markov chain with the set of states $Z^{1}$ and transition probabilities $p_{i, j}, i, j \in Z^{1}$ such that

$$
\begin{gathered}
p_{i, j}=p_{+, j-i} \text { and } \sum_{j} p_{+, j-i}(j-i)<0 \text { for all } i>d_{1}>0 \\
p_{i, j}=p_{-, j-i} \text { and } \sum_{j} p_{-, j-i}(j-i)>0 \text { for all } i<-d_{1}<0
\end{gathered}
$$

and

$$
p_{i, j}=0 \text { if }|j-i|>d_{2}
$$

with some positive constants $d_{1}, d_{2}$, is also ergodic and satisfies the conditions D.

These two examples are trivial. To get not trivial examples one can consider the almost homogeneous random walk in $Z_{+}^{\nu}$ satisfying some additional conditions (see [1], [4], and [5] for examples ).

Let $\xi_{t}(i), i \in X$, be a random walk in $X$ corresponding to our Markov chain starting at the point $i$.

Theorem 4.1.1 Kolmogorov inequality Let $V_{t}(i, j), i, j \in X, t \in Z_{+}$, be independent random vectors with the values in $R^{\mu}$, such that for any $i, j \in X$ the vectors $V_{t}(i, j), t \in Z_{+}$are identically distributed and

$$
\left\|V_{t}(i, j)\right\| \leq C_{0}<\infty \text { a.s }
$$

where $C_{0}>0$ does not depend on $i, j$. 
Then for any $i \in X$ there exists $c=c(i)>0$ such that for any $\delta>0$ and for any $T \in Z_{+}$

$$
P\left\{\sup _{\tau=1, \ldots, T}\left\|\sum_{n=0}^{t} V_{n}\left(\xi_{n}(i), \xi_{n+1}(i)\right)-E V_{n}\left(\xi_{n}(i), \xi_{n+1}(i)\right)\right\|>\delta T\right\} \leq \frac{c}{\delta^{2} T}
$$

To prove this theorem we shall need the following

Lemma 4.1.1 For any $i \in X$ there exist $c_{1}>0$ and $\alpha_{1}>0$ such that for any $n, m \in Z_{+}$

$$
\begin{aligned}
& \mid E\left(V_{n}\left(\xi_{n}(i), \xi_{n+1}(i)\right)-E V_{n}\left(\xi_{n}(i), \xi_{n+1}(i), V_{m}\left(\xi_{m}(i), \xi_{m+1}(i)\right)-\right.\right. \\
& \left.-E V_{m}\left(\xi_{m}(i), \xi_{m+1}(i)\right)\right) \mid \leq c_{1} e^{-\alpha_{1}|n-m|}
\end{aligned}
$$

where (.,.) is a scalar product in $R^{\mu}$,

$$
(x, y)=\sum_{j=1}^{\mu} x^{j} y^{j}, x, y \in R^{\mu}
$$

Proof of lemma 4.1.1

Let $i \in X$ be fixed, and

$$
V_{n}=V_{n}\left(\xi_{n}(i), \xi_{n+1}(i)\right), a_{n}=E V_{n}, n \in Z_{+} .
$$

For any $n, m \in Z_{+}, n>m$, one can easily get

$$
\begin{gathered}
E\left(V_{n}-a_{n}, V_{m}-a_{m}\right)=\sum_{i^{\prime}, i^{\prime \prime} \in X} p_{i, i^{\prime}}^{(m)} p_{i^{\prime}, i^{\prime \prime}} \times \\
\times \sum_{j^{\prime}, j^{\prime \prime} \in X}\left(p_{i^{\prime \prime}, j^{\prime}}^{(n-m-1)}-\pi\left(j^{\prime}\right)\right) p_{j^{\prime}, j^{\prime \prime}} \times \\
\times\left(E V_{m}\left(i^{\prime}, i^{\prime \prime}\right)-a_{m}, E V_{n}\left(j^{\prime}, j^{\prime \prime}\right)-a_{n}\right)
\end{gathered}
$$

From 76 by 73 it follows that 


$$
\begin{gathered}
\left|E\left(V_{n}-a_{n}, V_{m}-a_{m}\right)\right| \leq 4 C_{0}^{2} \sum_{i^{\prime}, j^{\prime} \in X} p_{i, i^{\prime}}^{(m+1)} \times \\
\cdot\left|p_{i^{\prime}, j^{\prime}}^{(n-m-1)}-\pi\left(j^{\prime}\right)\right|
\end{gathered}
$$

From ( 77), ( 71) and ( 72) one can easily get ( 75).

Lemma 4.1 .1 is proved.

\section{Proof of theorem 4.1.1}

Let $i \in X$ be fixed, $V_{n}=V_{n}\left(\xi_{n}(i), \xi_{n+1}(i)\right)$,

$$
a_{n}=E V_{n} \text { and } S_{n}=\sum_{j=0}^{n}\left(V_{j}-a_{j}\right), n \in Z_{+} .
$$

For given $\delta>0$ and $T \in Z_{+}$let us consider the following events

$$
A_{n}=\left\{\left\|S_{n+1}\right\|>\delta T,\left\|S_{0}\right\|<\delta T, \ldots,\left\|S_{n}\right\|<\delta T\right\}, n=0, \ldots, T .
$$

Let $I_{n}$ be an indicator of the event $A_{n}$. Then

$$
E\left\|S_{t}\right\|^{2} \geq \sum_{n=0}^{t-1}\left\|S_{n}\right\|^{2}-2 \sum_{n=o}^{t-1} \sum_{j=n+1}^{t-1}\left|E\left(S_{n} I_{n}, V_{l}-a_{j}\right)\right|=J_{1}(t)-J_{2}(t)
$$

Lemma 4.1.2 There exists $C>0$ such that for any $t$

$$
J_{2}(t) \leq C
$$

Proof. From (78) and (73) it follows that for any $h>0$ there exists $C_{1}>0$ such that

$$
\sum_{j=n+1}^{t-1}\left|E\left(S_{n} I_{n}, V_{l}-a_{j}\right)\right| \leq C_{1} E\left(\left|S_{n}\right| I_{n} \exp \left\{h \xi_{x}^{\Lambda}(n)\right\}\right)
$$

Note that due to ( 73$)$

$$
I_{n}=0 \text { a.s. for } n \leq \frac{\delta t}{2 C_{0}}
$$

Then from ( 73),( 80$)$ and ( 81$)$ we get 


$$
I_{2} \leq C_{1} \sum_{n=\theta t}^{t-1} n E\left(I_{n} \exp \left\{h \xi_{x}^{\Lambda}(n)\right\}\right)
$$

where $\theta=\frac{\delta}{2 C_{0}}$.

Consider the following event

$$
B_{n}=\left\{\xi_{x}^{\Lambda}(n)>\epsilon t\right\}
$$

Let $I_{B_{n}}$ be its indicator. Consider

$$
\begin{gathered}
J_{2}^{\prime}(t)=\sum_{n=\theta t}^{t-1} n E\left(I_{A_{n}} I_{n} \exp \left\{h \xi_{x}^{\Lambda}(n)\right\}\right) \\
J_{2}^{\prime \prime}(t)=\sum_{n=\theta t}^{t-1} n E\left(\left(1-I_{A_{n}}\right) I_{n} \exp \left\{h \xi_{x}^{\Lambda}(n)\right\}\right)
\end{gathered}
$$

For $J_{2}^{\prime \prime}(t)$ for small $\epsilon>0$ one can easily get

$$
J_{2}^{\prime \prime} \leq \sum_{n=\theta t}^{t-1} n E\left(I_{n} \exp \{\epsilon t\}\right) \leq C_{2} \exp (-\beta t)
$$

because of

$$
E\left(I_{n}\right) \leq C_{2}^{\prime} \exp \left(-\beta^{\prime} t\right)
$$

For $J_{2}^{\prime}$ we have

$$
J_{2}^{\prime} \leq \sum_{n=\theta t}^{t-1} n E\left(I_{A_{n}} \exp \left\{h \xi_{x}^{\Lambda}(n)\right\}\right) \leq C_{3} \exp (-\gamma t)
$$

because of

$$
E\left(\exp \left(h \xi_{x}^{\Lambda}(n)\right)\right)<\infty
$$

for small $h>0$.

From ( 82),( 83) and ( 84) it follows ( 79).

Lemma 4.1 .2 is proved.

From ( 78) using lemma 4.1.1 we get

$$
E\left\|S_{t}\right\|^{2} \geq \sum_{n=0}^{t-1} E\left\|S_{n}\right\|^{2} I_{n}-C
$$


where $c^{\prime}=c^{\prime}(x)>0$.

Let us note now that

$$
\sum_{n=0}^{t-1} E\left\|S_{n}\right\|^{2} I_{n} \geq \delta^{2} t^{2} P\left\{\max _{n=1, \ldots, t}\left\|S_{n}\right\|>\epsilon t\right\}
$$

From ( 85) and ( 86) we get

$$
P\left\{\max _{n=1, \ldots, t}\left\|S_{n}\right\|>\epsilon t\right\} \leq \frac{E\left\|S_{t}\right\|^{2}+C}{\epsilon^{2} t^{2}}
$$

Let us now estimate the value $E\left\|S_{t}\right\|^{2}$.

$$
E\left\|S_{t}\right\|^{2} \leq \sum_{j=1}^{t} E\left\|V_{j}-a_{j}\right\|^{2}+2 \sum_{j=1}^{t} \sum_{e=j+1}^{t}\left|E\left(V_{j}-a_{j}, V_{e}-a_{e}\right)\right|
$$

From ( 88 ) using ( 73 ) and lemma 4.1.1 we get

$$
E\left\|S_{t}\right\|^{2} \leq c^{\prime \prime} t
$$

where $c^{\prime \prime}=c^{\prime \prime}(x)>0$

From ( 87) and ( 89) we get ( 74)

The theorem 4.1 .1 is proved.

From the theorem 4.1 .1 by the ergodicity of the chain $\mathcal{L}_{\wedge}$ it easily follows

Theorem 4.1.2 Let the conditions of theorem 4.1 .1 be satisfied, and

$$
V=\sum_{i, j \in X} \pi_{i} p_{i, j} E V_{0}(i, j)
$$

Then for any $i \in X$ there exists $c=c(i)>0$ such that for any $\delta>0$ and for any $t \in Z_{+}$the following estimation holds

$$
P\left\{\sup _{\tau=1, \ldots, t}\left\|\sum_{n=0}^{\tau} V_{n}\left(\xi_{n}(i), \xi_{n+1}(i)\right)-V \tau\right\|>\delta t\right\} \leq \frac{c}{\delta^{2} t}
$$




\section{References}

[1] V.A.Malyshev. "Networks and Dynamical Systems". Adv. Appl. Probab., 25, pp.140-175, 1993.

[2] P.Dupuis and R.Ellis. "Large deviations for Markov Processes with discontinuous statistics,II: random walks". Probab. Theory Relat. Fields ,91, pp.153-194, 1992.

[3] P.Dupuis, H.Ishii and H.Mete Soner. "A viscosity solution approach to the asymptotic analysis of queueing systems". The Annals of Probability , 18, No.1, pp. 226-255. 1990.

[4] I.Ignatyuk, V.Malyshev. " Classification of Random Walks in $Z_{+}^{4}$ ". Selecta Mathematica (formerly Sovietica), Vol.12, No. 2, pp. 129-194, 1993.

[5] V.Malyshev, M.V.Menshikov. "Ergodicity, continuity and analyticity of countable Markov chains", Trans. Moscow Math. Soc. v.39, pp. 3- 48, 1979.

[6] V.Malyshev. "Asymptotic behaviour of stationary probabilities for two dimensional positive random walks", Siberian Math. Journal, v. 14. No. 1, pp. 156-169, 1972.

[7] A.Venttsell. "Limit theorems on Large Deviations for Markov stochastic processes". Kluwer Academic Press. 1990.

[8] J.-D. Deuschel, D.W.Stroock. Large Deviations. Acad.Press,Inc.1989.

[9] R.S.Ellis. "Entropy, Large Deviations, and Statistical Mechanics." Springer-Verlag. 1985.

[10] R.L.Dobrushin. "Analyticity of correlation functions in one-dimensional classical systems with slowly decreasing potentials." Comm.Math.Phis., vol.32, 1973, 269-289.

[11] M.Campanino, D.Capocaccia, E.Olivieri. "Analyticity for onedimensional systems with long-range superstable interactions. J.Statist. Phis., vol.33, No.2, 1983, 437-476. 
Unité de Recherche INRIA Rocquencourt

Domaine de Voluceau - Rocquencourt - B.P. 105 - 78153 LE CHESNAY Cedex (France)

Unite de Recherche INRIA Lorraine Technopôle de Nancy-Brabois-Campus Scientifique

615. ruc du Jardin Botanique - B.P. 101 - \$4602 VILLERS LES NA:NCY Cedex (France)

Unité de Recherche INRIA Rennes IRISA. Campus Universitaire de Beaulieu 35042 RlENNES Cedex (France)

Unite de Recherche INRIA Rhône-Alpes 46, avenue Félix Viallet - 38031 GRF.NOBI.F. Cedex (France)

binite de Recherche INRIA Sophia Antipolis 2014. route des Lucioles - B.P. 93 - 06902 SOPHIA ANTIPOLIS Cedex (France)

\section{EDITEUR}

INRIA - Domaine de Voluceau - Rocquencourt - B.P. 105 - 78153 LE CHESNAY Cedex (France) 